\title{
Die Berufspassage in das Unternehmertum
}

\section{Theoretische und empirische Befunde zum sozialen Prozeß von Firmengründungen}

\section{Dieter Bögenhold}

Institut für Wirtschafts- und Sozialwissenschaften, Universität Münster, Kleimannstraße 5, D-4400 Münster

Zusammenfassung: Der Aufsatz beschäftigt sich zunächst theoretisch und sekundär-statistisch mit der beruflichen Ein- und Austrittsmobilität bei der Wirtschaftskategorie der Selbständigen und konstatiert diesbezüglich eine relativ beachtliche individuell-personelle Fluktuation, welche in den ..großen" Gesellschaftsentwürfen früherer Zeit entweder kaum registriert oder bestritten worden war. Daran anschließend wird gefragt, wie der Schritt in die selbständige Erwerbsarbeit auf der Akteursebene operationalisiert wird. Diesbezüglich nimmt der Beitrag Bezug auf Ergebnisse einer empirischen Pilot-Untersuchung, die sich mit den Instrumentarien beschäftigt, welche in dem sozialen Prozeß der Konstitution einer Firma eingesetzt werden. Hier wird deutlich, daß neben den in unterschiedlicher Komposition relevanten Ressourcen Geld und Wissen der Rolle der Sozialkontakte eine Schlüsselfunktion zukommt.

Was Joseph Schumpeter vor mehr als einem halben Jahrhundert formulierte, nämlich daß „,es erstaunlich, aber eine Tatsache (ist), daß die Art, wie man Unternehmer wird, bisher so wenig untersucht wurde" (Schumpeter, 1985 [1927], S. 168), gilt noch heute gleichermaßen. Es gibt kaum Informationen, unter welchen Umständen, mit welchen Zielen und unter Mobilisierung welcher Instrumente einzelne Menschen Unternehmer werden. Der vorliegende Beitrag widmet sich dieser Frage und stellt theoretische und statistische Befunde zusammen, die um die ersten Ergebnisse einer empirischen Pilotstudie ergänzt und modifiziert werden, die den beruflichen Weg in das selbständige Unternehmertum ${ }^{1}$ zum Thema hatte. Erklärtes Ziel ist es, in Richtung der von Schumpeter diagnostizierten terra incognita aufzubrechen und erste Schritte zu wagen, damit die Forschungslücke zumindest ein Stück weit mit Leben gefüllt wird, alles in der Hoffnung, daß weitere soziologische Arbeiten die Spur aufnehmen werden.

Die nachstehenden Ausführungen gliedern sich folgendermaßen: Zunächst wird in die Thematik „Small Business und selbständige Erwerbstätig-

\footnotetext{
Schumpeter faßte mit seiner Begrifflichkeit des Unternehmers sowohl angestellte Manager wie auch selbständige Eigentümer - Unternehmer. Zu praktischen und theoretischen Unterschieden zwischen selbständigen und angestellten Unternehmern vgl. T. Veblen (1924), J. Burnham (1941) und H. Hartmann (1959). In der vorliegenden Untersuchung stehen nur die selbständig erwerbstätigen Wirtschaftsindividuen zur Diskussion.
}

keit" eingeführt und darauf aufbauend dann die im Vordergrund stehende Frage nach der intragenerationellen Mobilität in die wirtschaftliche Selbständigkeit entwickelt: Die verfügbaren statistisch quantifizierbaren Angaben über die berufliche Fluktuation in das Unternehmertum informieren uns (bestenfalls) darüber, $d a \beta$ in einem bestimmten Zeitraum so und so viele Menschen aus den und den beruflichen Herkunftsbereichen in die Selbständigkeit wechseln (oder letztere eben verlassen). Aber neben diesem Faktum, daß es so ist, wissen wir eigentlich nicht, wie dieses auf individueller Ebene im Einzelfall realisiert wird. Mit anderen Worten: Nicht nur die jeweiligen Endergebnisse gesellschaftlicher Bewegung interessieren, sondern ebenso die sozialen Bausteine, die als Elemente die gesellschaftliche Motorik erst in Gang setzen und halten. Damit ist hier die Frage nach dem modus operandi des Selbständigmachens angesprochen. In der empirischen Aufarbeitung einer begrenzten Zahl von Firmengründungen wird diese Frage ernstgenommen und stellvertretend versucht, die einzelnen Schritte und Muster der beruflichen Mobilität des "Selbständigmachens" en détail zu rekonstruieren. Im Vordergrund des Interesses steht hierbei die Erarbeitung der Kombination von Motiven, strategischen Konzeptionen und ökonomischen, kulturellen und sozialen Ressourcen, welche in dem sozialen Prozeß der Gründung einer wirtschaftlich-selbständigen Existenz eingesetzt werden. Insgesamt wird hier deutlich, daß es differente soziale „Logiken“ der Mobilität in das Unternehmertum gibt und daß der Existenz von sozialen Netzwerken im Prozeß der Gründung 
einer Firma eine große, praktisch und theoretisch bisher übersehene Rolle zukommt.

\section{Die Selbständigen in der Sozialstruktur}

Nachdem die Zahl der Selbständigen über Jahrzehnte rückläufig war, scheint sie sich in der Bundesrepublik etwa seit Beginn der 80er Jahre stabilisiert zu haben, freilich auf historisch vergleichsweise niedrigem Niveau. Als Selbständige gelten diejenigen Erwerbstätigen, die nicht abhängig beschäftigt sind und stattdessen auf eigene Rechnung Produkte oder Dienstleistungen erstellen, die sie auf dem Markt verkaufen. Dieser definitorische Hauptnenner beinhaltet ein enorm breites Spektrum von je spezifischen sozioökonomischen Lagen. In dem „,sozialen Kunterbunt“ (Th. Geiger), das die Selbständigen repräsentieren, finden sich die klassischen Unternehmerkapitalisten mit teilweise beträchtlichen Produktionsmitteln und groBem Betriebsvermögen ebenso wie die Unternehmer, insbesondere Kleinunternehmer, bei denen das Betriebsvermögen eine untergeordnete Rolle spielt, also - in Marx'schen Begriffen - die kapitalistischen und die einfachen Warenproduzenten. Die einen betreiben ihren Produktionsprozeß mit zahlreichen ,angekauften“ Arbeitskräften, während andere nur wenige Lohnarbeitskräfte beschäftigen oder auch völlig ohne fremde Arbeitskräfte auskommen. Letzteres ist naturgemäß bei den kleineren und kleinsten Firmen der Fall, wo entweder ein Team mit gemeinsamen Besitzrechten am Betriebsvermögen oder der Firmeninhaber als einzelner die anfallenden Arbeiten und $\mathrm{Ge}$ schäfte erledigt. Außerdem findet sich im Kleingewerbe oft der nicht unmittelbar entlohnte Einsatz von Familienarbeitskräften, der sogenannten mithelfenden Familienangehörigen.

Der Anteil der Selbständigen, die im Besitz von Groß- und Größtunternehmen sind, ist verschwindend gering. Der überragende Teil (ca. 98 Prozent) hat Firmen, die als kleine und mittlere Unternehmen gelten und definiert sind als Firmen mit bis $\mathrm{zu} 500$ Beschäftigten. Das Gros der letztgenannten Unternehmer verteilt sich allerdings auf kleine und kleinste Unternehmungen und 46 Prozent aller Selbständigen betreiben ihre Geschäfte ohne den Einsatz von festen abhängig Beschäftigten $^{2}$. Für die meisten Selbständigen gilt, daß sie

\footnotetext{
${ }^{2}$ Eigene Berechnungen nach Statistisches Bundesamt:
} Fachserie 1, Reihe 4.1.1. mit ihren kleinen Firmen jene Märkte okkupieren, welche sich für eine standardisierte Massenproduktion nicht (bzw. nur schlecht) anbieten. Daß es die Selbständigen als Wirtschaftskategorie auch heute noch gibt, muß angesichts der Tatsache verwundern, daß sie über viele Jahre Prophezeiungen ausgesetzt waren, die ihnen den baldigen Tod voraussagten. Diese Prophezeiungen waren in der Hypothese begründet, daß in der unaufhaltsamen Tendenz zum Großbetrieb die Klein- und Kleinstunternehmen konkurrenzmäßig unterlegen seien und demzufolge nach und nach aussterben würden; und in den Großbetrieben selber, so glaubte man, würden die selbständigen Unternehmer im Zuge des Aufkommens der Aktiengesellschaften und der einsetzenden Trennung von Eigentum und Kontrolle zunehmend verschwinden. Die Anhänger dieser Hypothese, nämlich daß die Kleinbetriebe ,zerrieben“ (Marx) würden, fanden sich durchgehend in einem breiten wissenschaftspolitischen Spektrum. Schließlich fand sie auch zunächst empirische Bestätigung, besonders in der zweiten Hälfte des letzten Jahrhunderts, als zahlreiche Kleingewerbezweige liquidiert wurden und der Verein für Socialpolitik sich auf mehreren Kongressen beispielsweise der Lage des Handwerks annahm. In diesem Jahrhundert setzte sich der Selbständigenrückgang relativ bruchlos bis in die "neuere" Zeit fort. So waren bis Ende der 70er Jahre stetige Verluste zu verzeichnen. Doch es sieht nun so aus, als ob die Talfahrt der Selbständigen ihr Ende erreicht habe und daß sie sich auf ihrem jetzigen Niveau zumindest konsolidiert haben.

Die Tabelle (1) gibt über die relative und absolute Entwicklung der Selbständigenzahlen seit 1950 Auskunft. Zunächst verdeutlicht sich hier, daß das „Selbständigensterben“ offenbar gestoppt ist. Für die letzten Jahre wird sogar eine leichte Zunahme der Selbständigen ausgewiesen. Berücksichtigt man aber auch die mithelfenden Familienangehörigen, so zeigt sich, daß sich beide Gruppen zusammen anzahlmäßig auf einem historisch sehr niedrigen Stand befinden. Nun sagt die Betrachtung der absoluten Zahl der Selbständigen noch nichts über deren Anteil an der Gesamtzahl der Erwerbstätigen aus. Dazu bedarf es des Blickes auf die (errechneten) Quoten für die Selbständigen (gemessen an allen Erwerbstätigen). Die Selbständigenquote I berücksichtigt dabei lediglich die Selbständigen im engeren Sinne, während bei der Selbständigenquote II die ,mithelfenden Familienangehörigen“ hineingerechnet sind. Es zeigt sich dabei, daß die absolute Abnahme der Selbständigen von 
Tabelle 1 Selbständige und Selbständigenquoten in der Bundesrepublik Deutschland insgesamt und nach Wirtschaftsbereichen in den Jahren 1950-1987 (in 1000 bzw. v.H.).

\begin{tabular}{lllllllllllll}
\hline Jahr & $\begin{array}{l}\text { Selb- } \\
\text { ständige }\end{array}$ & $\begin{array}{l}\text { Mithelfende } \\
\text { Familien- } \\
\text { angehörige }\end{array}$ & $\begin{array}{l}\text { Quoten } \\
\text { insgesamt }\end{array}$ & & & & & $\begin{array}{l}\text { Land- }+ \\
\text { Forstwirt- } \\
\text { schaft }\end{array}$ & $\begin{array}{l}\text { Produzie- } \\
\text { rendes } \\
\text { Gewerbe }\end{array}$ & $\begin{array}{l}\text { Handel + } \\
\text { Verkehr }\end{array}$ & $\begin{array}{l}\text { Sonstige } \\
\text { Wirtschafts- } \\
\text { bereiche } \\
\text { (Dienstleist.) } \\
\text { II }\end{array}$ \\
\hline 1950 & 3200 & 3125 & 16,0 & 31,6 & 25,6 & 80,0 & 10,6 & 12,5 & 21,9 & 27,2 & 9,9 & 12,7 \\
1960 & 3240 & 2560 & 12,8 & 22,9 & 32,5 & 84,9 & 6,8 & 8,7 & 16,2 & 21,5 & 9,4 & 13,0 \\
1970 & 2690 & 1732 & 10,1 & 16,6 & 33,9 & 87,0 & 5,0 & 6,1 & 14,3 & 18,7 & 9,0 & 11,7 \\
1975 & 2445 & 1351 & 9,5 & 14,7 & 35,1 & 87,0 & 4,9 & 6,0 & 13,3 & 16,5 & 8,1 & 10,1 \\
1977 & 2379 & 1139 & 9,3 & 13,8 & 35,4 & 85,5 & 4,9 & 5,9 & 13,3 & 15,7 & 8,0 & 9,5 \\
1980 & 2360 & 959 & 9,0 & 12,6 & 35,7 & 83,0 & 4,8 & 5,6 & 12,8 & 14,8 & 7,8 & 9,0 \\
1982 & 2370 & 903 & 9,2 & 12,7 & 36,4 & 82,6 & 5,0 & 5,8 & 13,1 & 15,0 & 8,0 & 9,0 \\
1983 & 2393 & 881 & 9,4 & 12,9 & 36,4 & 82,2 & 5,2 & 5,9 & 13,5 & 15,3 & 8,1 & 9,1 \\
1984 & 2405 & 861 & 9,5 & 12,9 & 36,2 & 81,2 & 5,2 & 5,9 & 13,7 & 15,4 & 8,2 & 9,1 \\
$1985^{*}$ & 2415 & 851 & 9,5 & 12,8 & 36,2 & 81,5 & 5,2 & 5,9 & 13,8 & 15,4 & 8,1 & 9,1 \\
$1986^{*}$ & 2422 & 845 & 9,4 & 12,7 & 36,2 & 81,5 & 5,2 & 5,8 & 13,7 & 15,4 & 8,1 & 9,1 \\
$1987^{*}$ & 2426 & 838 & 9,3 & 12,6 & 36,2 & 81,5 & 5,2 & 5,8 & 13,7 & 15,3 & 8,1 & 9,0 \\
\hline
\end{tabular}

* = vorläufiges Ergebnis

$\mathrm{I}=$ Anteil der Selbständigen an den Erwerbstätigen in v.H.

II = Anteil der Selbständigen und mithelfenden Familienangehörigen an den Erwerbstätigen insgesamt in v. $\mathrm{H}$.

Quelle: Statistisches Jahrbuch (verschiedene Jahrgänge) und eigene Berechnungen danach

einem Sinken der Selbständigenquote begleitet wurde. Die starken Veränderungen in den 50er Jahren sind insbesondere auf die überproportional gestiegenen absoluten Zahlen der abhängig Beschäftigten zurückzuführen. Auffällig ist auch, daß die Quoten sich in den 70er Jahren in deutlich gedrosseltem Tempo verringerten.

Die Differenzierung nach den einzelnen Wirtschaftssektoren ergibt interessanterweise, daß die jeweiligen Wirtschaftsbereiche offenbar eine spezifische Selbständigenrate aufweisen, die sich teilweise beträchtlich von der durchschnittlichen Gesamtrate unterscheidet. Die niedrigsten Selbständigenquoten (I und II) weist das produzierende Gewerbe auf, während in der Land- und Forstwirtschaft die höchsten vorliegen. Und obwohl die Land- und Forstwirtschaft seit 1950 mehr als 70 Prozent ihrer Erwerbstätigen eingebüßt hat, ist sie nach wie vor von Selbständigen und ihren ,mithelfenden Familienangehörigen" dominiert. Das verdeutlicht auch, daß die strukturellen volkswirtschaftlichen Veränderungen und Verschiebungen (Stichwort: Tertiärisierung) zwar deutliche Spuren in bezug auf die Bedeutung und das Gewicht der einzelnen Sektoren hinterlassen haben, jedoch kaum nennenswerte Einflüsse auf deren inneres Erwerbstätigengefüge hatten.

Die Wirtschaftskategorie der Selbständigen ist nicht nur unter dem Gesichtspunkt ihrer konkreten wirtschaftlichen Tätigkeiten und der jeweiligen Betriebsgrößen der Wirtschaftsunternehmen ausgesprochen heterogen, sondern auch im Hinblick auf die vorzufindenden Einkommenslagen (vgl. Bögenhold 1987a, S. 18f.). Die Spanne reicht von gesellschaftlichen Spitzeneinkommen auf der einen Seite bis zu armutsnahen Einkommen auf der anderen. Gerade das aber spricht bereits dagegen, die Kategorie der Selbständigen in dem Begriff des Mittelstandes aufgehen zu lassen bzw. sie mit „Mittelstand“ oder ,-klasse" zu übersetzen. Der soziologisch problematische Terminus des Mittelstandes, den Theodor Geiger zu Recht als ,Verlegenheitsbegriff“ (Geiger 1962, S. 235) oder „Unbegriff" (Geiger 1967, S. 128) bezeichnet hat, weist darauf hin, daß die Selbständigen unter sozialstrukturellem Gesichtspunkt und klassen- und/oder 
schichtungstheoretischen Fragestellungen ein „Problemkind" der Soziologie sind bzw. waren, das zudem nicht gerade mit Aufmerksamkeit verwöhnt wurde. So gehören sie einerseits nicht $\mathrm{zu}$ den abhängig Beschäftigten, noch paßt für die meisten von ihnen der Begriff der Kapitalisten mit seinem traditionellen semantischen Gehalt. Jene Autoren, die davon ausgingen, die Erwerbsgruppierung der Selbständigen würde im Zuge von sozioökonomischen Polarisierungsprozessen allmählich verschwinden, schenkten den Selbständigen - und besonders den „kleinen“ - überhaupt keine Aufmerksamkeit; für andere war es stets eine ambivalente sozialstrukturelle „Restkatego-

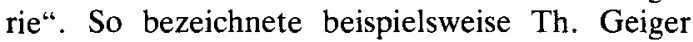
„die Gesamtheit der Leute, von denen ich weder behaupten kann, sie hätten kein Kapital, noch sie seien Kapitalisten“, als den "Posten ,Sonstige““" (Geiger, 1962, S. 235). Die Heterogenität der Selbständigen führte zwei britische Autoren zu der illustrativ-begrifflichen Unterscheidung zwischen einer „haute“ und einer „petite Bourgeoisie“, wobei sie letztere schon im Untertitel eines Buches als „uneasy stratum" auswiesen (Bechhofer/Elliott (eds.), 1981).

Während die Volks- und Betriebswirtschaftswissenschaftler stets in dem thematischen Bereich der „kleinen und mittleren Unternehmen“, also dem Feld, in dem die weitaus meisten Selbständigen mit ihren wirtschaftlichen Unternehmungen tätig sind, geforscht $z u$ haben, muß das Verhältnis der deutschen Nachkriegssoziologie zu den selbständigen Unternehmern nachgerade als unterkühlt bezeichnet werden. Das Feld ist mit „kompakten" Ausarbeitungen dünn gesät (Hartmann, 1959; Hartmann et al. 1968; Biermann, 1971; Urbat, 1974; Bögenhold, 1985; Bögenhold, 1987a). Zwar werden die Selbständigen auch von der anglo-amerikanischen Soziologie nicht gerade mit üppiger Aufmerksamkeit verwöhnt, doch findet dort zumindest ,auf kleiner Flamme“ eine Diskussion über "Small Business" und „Entrepreneurship“ statt. Allerdings kann sich das hierzulande bisher relativ unbeschriebene Blatt insofern schnell wenden, als die Selbständigen nicht nur unter sozialstrukturellen Aspekten von der Soziologie stärker beachtet werden müßten, sondern als vor allem im Zuge eines wachsenden Interesses an soziologischen Spekulationen über die „Zukunft der Arbeit", „, neue Produktionskonzepte“ und „Alternativen zur Massenproduktion" derzeit auch von der Arbeitsmarktund der Industrie- und Betriebssoziologie Bedarf nach basalen, originär soziologischen Arbeiten über Kleinbetriebe und Kleinunternehmer angemeldet wird.

\section{Ein- und Austrittsmobilität bei den Selbständigen}

Die Daten über den absoluten und relativen Umfang der Selbständigen und dessen Veränderung können insofern nur einen knappen Überblick über die Entwicklungen bei den Selbständigen geben, als diese Daten lediglich die Summe vielschichtiger sozioökonomischer Prozesse sind. Da es sich um Saldozahlen handelt, bleibt die ,hinter" den Daten sich vollziehende Bewegung weitgehend verborgen. So sagt die Selbständigenstatistik über die Nettofluktuation zwischen den Selbständigen und den anderen Erwerbspersonengruppierungen zunächst nichts aus.

Für die Arbeitsmarkt- und Berufssoziologie wie auch für klassen- und schichtungssoziologische Diskussionen ist aber gerade die berufliche Mobilität von besonderem Interesse. Die Leitfrage lautet dabei, inwieweit die Sozialstruktur als Gefüge gesellschaftlicher Positionen individuell-personal ,aufgemischt" wird, d. h. inwieweit die Positionen von stetig wechselnden Personen eingenommen werden. Schumpeter drückte diese Perspektive in seiner Analogie aus, daß jede Klasse ,während der Dauer ihres Kollektivlebens ... einem Hotel oder einem Omnibus (gleiche), die zwar immer besetzt sind, aber von immer anderen Leuten" (Schumpeter 1953, S. 171). In den 50er und 60er Jahren waren es unter anderem Dahrendorf (1957) und Schelsky (1968, bes. S. 428f.), die auf die soziale Mobilität als analytischen Schlüssel hinwiesen, während die Soziologie der Sozialstruktur in jüngeren Jahren diese Fragen in Termini von Individualisierungsprozessen angeht (Beck 1986, Zapf et al. 1987). Dadurch daß die Menschen heute wesentlich häufiger als in früheren Jahrzehnten räumlich, beruflich und sozial „umgetopft" werden, kann der Effekt entstehen, daß das Bild der Sozialstruktur mit seinem (ungleichen) Gefüge verschieden privilegierter Positionen weitestgehend stabil geblieben ist, gleichzeitig aber die Zuordnung und Verteilung der (einzelnen) Personen auf (gesellschaftliche) Positionen weniger vorhersehbar und reglementiert ist. Was in den verschiedenen Arbeiten mit gesellschaftstheoretischen Schlußfolgerungen thematisiert wird, ist stets auf die empirische Untermauerung mit sozialstrukturellen Daten angewiesen. So muß die empirisch ausgerichtete, originär Daten erhebende bzw. auswertende neuere Mobilitätsforschung in diesem Zusammenhang einen zentralen Stellenwert haben. Für Großbritannien sind es u. a. die Arbeiten von Goldthorpe (1980) und G. Payne (1987) und Goldthorpe/Payne (1986), die Interesse beanspruchen dürfen, während für die Bundesrepublik die Studien von K. U. Mayer (1977) und W. Müller (1978) aus den 70er Jahren und die Ergebnisse aus ,,jüngeren Jahren" von K. U. Mayer und Kollegen über die Berufsverläufe verschiedener Geburtskohorten (vgl. aus der Vielzahl von inzwischen vorliegenden Beiträgen nur K. U. Mayer/Carroll, 1987; Blossfeld 1986; Carroly/Mayer 1986 ) interessante Hinweise geben (international verglei- 
chend vgl. außerdem: Müller 1986; Müller/Goldthorpe 1988).

Im vorliegenden Zusammenhang richtet sich das Interesse lediglich auf einen kleinen Ausschnitt der beruflichen Mobilität. Mit anderen Worten: Es sollen keine generellen mobilitätssoziologischen Betrachtungen mit weitreichenden gesellschaftstheoretischen Spekulationen angestellt werden, sondern statt dessen wird der Blick nur durch einen schmalen Schlitz geworfen, nämlich auf die Ein- und Austrittsmobilität bei den Selbständigen. Das ist also die Frage, inwieweit die Wirtschaftskategorie der Selbständigen sich weitestgehend selbst rekrutiert bzw. intergenerationell perpetuiert oder inwieweit sie personell (inter- und intragenerationell) stets neu „aufgemischt" wird. Empirisch ließ sich zeigen (vgl. K. U. Mayer, 1977), daß die Selbständigen relativ niedrige Vererbungseffekte aufweisen und daß bei ihnen beträchtliche intergenerationelle $\mathrm{Zu}$ - und Abwanderungsströme vorliegen. Eine Ausnahme stellten lediglich die Selbständigen in der Landwirtschaft dar, wo bekanntlich insgesamt starke Selbständigenverluste zu verzeichnen waren, der Wirtschaftsbereich sich aber gleichzeitig gegenüber möglichen Einsteigern aus nichtlandwirtschaftlichen Bereichen als relativ abgeschlossen zeigte. Zum überragenden Teil rekrutieren sich die Landwirte intergenerationell aus Kindern von Landwirten. Dieser Vererbungseffekt war bei den Selbständigen in den übrigen Wirtschaftsbereichen hingegen nur von untergeordneter Bedeutung.

Stellten diese Ergebnisse auf die intergenerationelle Mobilität ab (und dabei nur auf die „Berufe“" von Vätern und Söhnen), so beziehen sich „,neuere" Arbeiten auf die intragenerationelle Mobilität. Beispielsweise wurden in der Untersuchung „Lebensläufe und Wohlfahrtsentwicklung" (K. U. Mayer et al.) die Berufsverläufe dreier Geburtskohorten von Frauen und Männern (1929-31, 1939-41 und 1949-51) in den Jahren 1981 und 1983 repräsentativ erhoben. Dabei verdeutlicht sich, $\mathrm{da}$ in der Wirtschaftskategorie der Selbständigen überdurchschnittliche Fluktuationsströme vonstatten gehen, wobei auch hier wieder die Landwirtschaft einen Sonderfall abgibt (Mosakowski/Carroll, 1985). Die Tatsache, daß die Kategorie der Selbständigen für Ein- und Austritte gegenüber den anderen Erwerbsgruppierungen „offener“ erscheint und daß die Selbständigen ständig „frisches Blut" erhalten, bedeutet im Umkehrschluß nicht, $\mathrm{daB}$ sich auch ein vergleichsweiser hoher Anteil von Arbeitslosen, Arbeitern und Angestellten selbständig machen kann (aus Gründen der Quantitäten).
Ebenso deuten andere statistische Befunde auf die überdurchschnittliche Mobilität bei den Selbständigen hin. Eine Auswertung von Daten über die berufliche Umschichtung innerhalb eines Jahres, die seitens des Statistischen Bundesamtes durchgeführt wurde (H. L. Mayer 1983, S. 782ff.), zeigte für den Fall der Selbständigen, daß die individuelle Verbleibquote nur bei 85 Prozent lag (bzw. bei 81 Prozent inklusive mithelfende Familienangehörige; vgl. differenziertere Interpretationen dieser Daten bei Bögenhold, 1987b). Da sich im Saldo zwischen den Erhebungszeitpunkten nur geringfügige Bruttoveränderungen ergaben, zeigte sich damit, daß die Zugänge ähnlich groß wie die Abgänge waren. Auch die ersten Ergebnisse der verschiedenen Erhebungswellen des „Sozio-ökonomischen Panel", das vom Deutschen Institut für Wirtschaftsforschung (DIW) zusammen mit dem Sonderforschungsbereich 3 ,Mikroanalytische Grundlagen der Gesellschaftspolitik" durchgeführt wird (vgl. zur Konzeption U. Hanefeld, 1987), verdeutlichen die Ein- und Austrittsmobilität bei der Kategorie der Selbständigen (vgl. Schupp, 1988). Da sich im Saldo zwischen den Erhebungszeitpunkten auch hier nur geringfügige Bruttoveränderungen ergaben, zeigt sich, daß die Zugänge ähnlich groß wie die Abgänge waren. Neue Ergebnisse der ersten vier Wellen des „Sozio-ökonomischen Panels“ verdeutlichen die Ein- und Austrittsmobilität besonders prägnant. So waren beispielsweise von den Selbständigen des Jahres 1987 nur 72,3\% bereits im Jahre 1984 selbständig. Mit anderen Worten: Von den 100 Prozent im Jahre 1987 waren mehr als ein Viertel erst im Verlauf der letzten drei Jahre „zugestiegen“. Wenn die umgekehrte Perspektive eingenommen wird, nämlich was aus den Selbständigen des Jahres 1984 in den folgenden Jahren wurde, ergibt sich ein ähnliches Bild: Im Jahre 1987 waren nur noch $69,1 \%$ selbständig erwerbstätig. Demnach müssen ständig viele die Selbständigkeit verlassen, während gleichzeitig Menschen in die Selbständigkeit drängen. Die Aufschlüsselung sowohl der Zustrom- wie der Abstromquote zeigt keine exklusiven Wege in die bzw. aus der Selbständigkeit. Insgesamt vollziehen sich hier permanente Prozesse „sozialen Auftriebs" und „sozialer Deklassierung“" (Schumpeter 1964).

\section{Heterogenität der Einstiegsmuster}

Zunächst einmal ist es erstaunlich, daß es den beruflichen Mobilitätskanal in das selbständige Unternehmertum gibt. Zwar ist dieses keine Hauptverkehrsstraße gesellschaftlicher Mobilität, 
doch immerhin scheint es eine durchaus passabel frequentierte Nebenverkehrsstrecke darzustellen. Auf ihr verlassen also nicht nur ständig Personen das Unternehmertum, sondern werden ihm ebenso auch zugeführt, und zwar keineswegs nur durch Erbschaft.

Erstaunlich ist es insofern, als in der sozialwissenschaftlichen Literatur ja über lange Zeit der Gedanke einer eher strikten Trennung der beiden großen Hauptklassen im Vordergrund stand, nämlich die der Produktionsmittelbesitzer hier und die der Lohnabhängigen dort. Der Übergang in die selbständige Erwerbstätigkeit war weder bei Marx noch bei Weber oder schließlich Theodor Geiger empirisch registriert und gesellschaftstheoretisch berücksichtigt. In den „großen“ Entwürfen fand sich stattdessen nur die Betonung der gesamtgesellschaftlich-säkularen Entwicklung in umgekehrter Richtung, nämlich der Minimierung der selbständigen Erwerbsarbeit und der sich parallel ausdehnenden Lohnarbeit bzw. abhängigen Beschäftigung. Das war zeitgeschichtlich durchaus verständlich, nicht zuletzt weil Arbeiter nicht in der Lage waren, sich notwendiges Sparkapital anzulegen (vgl. z. B. F. A. Lange, 1865, S. 85). Nur vereinzelt gab es Hinweise, daß der soziale „Aufschwung von unten“ (Michels 1934, S. 64) in seltenen Fällen auch für Arbeiter möglich war, die dann von „Lohnsklaven“ zu Unternehmern wurden (Mill, 1848, S. 150, vgl. auch Fröbel, 1874, S. 128). Dieses waren jedoch eher Ausnahmen, was angesichts des aufkommenden Konkurrenzkapitalismus und der Bedrohung vieler kleingewerblicher Branchen nicht verwunderlich war. Erst viel später finden sich verstreute Belege in der Literatur, die auch den gegenläufigen Prozeß der (gesellschaftlich eher unterirdischen) Strömung in das (zumeist kleine) Unternehmertum konzedierten. So waren sowohl in Teilen des Handwerks wie des Handels in Zeiten hoher und anhaltender Arbeitslosigkeit Tendenzen festgestellt worden, daß vermehrt selbständige Klein- und Kleinstbetriebe gegründet wurden. Eine Arbeit über das Handwerk in Deutschland bezeichnet dieses als „Flucht in die Selbständigkeit" und als „unvermeidlichen Reaktionsvorgang“, der von einém Teil der „,arbeitslosen Gesellen und Facharbeiter“ beschritten wird. Dabei tritt dann der circulus vitiosus auf, daß der Konkurrenzkampf schärfer wird und die Lebensund Arbeitsbedingungen sich weiter verschlechtern (Wernet 1952, S. 276f., für den Handel vgl. Haupt (Hg.), 1985, S. 32f.).

Die zuvor referierten empirischen Befunde über die Ein- und Ausstiegsmobilität bei der Wirtschaftskategorie der Selbständigen verdeutlichen ja recht prägnant, daß die ,Teufelsmühle des Marktes" (Polanyi, 1978) die Gesellschaft eben nicht (bzw. nicht nur) in die große Gruppe der Lohnabhängigen hier und die kleinere der mächtigen Unternehmer dort zermahlen hat, sondern daß sie immer wieder in unterschiedlichen Graden auch neue Formen der erwerbstätigen Nichtlohn- arbeit produziert. Insgesamt sind die sozialen $\mathrm{Zu}$ gangsmuster in die Selbständigkeit unterschiedlich, besonders was die einzelnen biographischen Ausgangspunkte und die konkreten Motive anbelangt. Ist es angesichts der Heterogenität der sozialen Lagen der Selbständigen nicht unproblematisch, pauschal von den Selbständigen zu sprechen, so gilt dieses ebenso für die Formulierung von den Gründern, denn dabei werden die Verschiedenheiten der „Einstiegslogiken“ nicht berücksichtigt.

Einerseits läßt sich feststellen, daß der Druck auf dem Arbeitsmarkt vermittelt durch die langanhaltend vergleichsweise hohe Arbeitslosigkeit in relevanten Teilarbeitsmärkten verstärkt Menschen stimuliert, sich in Richtung einer selbständigen Erwerbsarbeit zu orientieren. Dadurch fungiert der Weg in die Selbständigkeit als kleines Ventil des Arbeitsmarktes. Eine Studie über sogenannte Existenzgründungen, die vom IFO-Institut (Weitzel, 1986) durchgeführt wurde, zeigte, da $\beta$ unter den Gründern die Zahl derer, die vorher arbeitslos waren, enorm gestiegen ist. So waren von den dort erfaßten Gründern im Jahre 1981 neun Prozent vorher arbeitslos gewesen (im Durchschnitt aller dort erfaßten Wirtschaftsbereiche), während es bei denen des Jahres 1983 schon doppelt so viele waren. In einzelnen Wirtschaftsbereichen liegt der Anteil der Gründungen aus der Arbeitslosigkeit noch weit darüber. Beispielsweise hat er sich im Handwerk von 10 auf 24 Prozent erhöht. Mit anderen Worten: Etwa jede vierte Gründung im Handwerk erfolgte aus der Arbeitslosigkeit heraus. Interessant ist ferner, daß bei den Gründern, die zuvor einen (abhängigen) Arbeitsplatz hatten, sich die Beurteilung ihrer bisherigen Beschäftigung nach deren Stabilität (sicher - unsicher) zwischen 1981 und 1983 ebenfalls veränderte. Bezeichneten noch 1981 etwa zwei Drittel von ihnen ihren bisherigen Arbeitsplatz als sicher, so waren es bei denen des Jahres 1983 nur noch 57 Prozent (Weitzel 1986, S. 71). Arbeitsmarktstudien über verschiedene akademische Teilarbeitsmärkte deuten in ähnliche Richtung, nämlich daß zunehmende Beschäftigungsrisiken den Zustrom in die Selbständigkeit forcieren. Das zeigte sich für die Absolventen von geisteswissenschaftlichen und Lehramts-Studiengängen wie für weite Teile der Hochschulabsolventen generell (vgl. v. a. einzelne Beiträge in Kaiser/ Nuthmann/Stegmann, Hrsg., 1985).

Andererseits gibt es neben diesen solchermaßen in die Selbständigkeit Geschobenen seit eh und je Menschen, die sich primär in Richtung der selbständigen Erwerbsarbeit orientieren, weil sie hier 
einen sozialen Aufstiegsweg sehen und/oder ein Reich der Autonomie und Selbstverwirklichung ausmachen. Die Gründung eines kleinen Unternehmens erscheint gerade auch für Arbeiter als traditioneller Aufstiegsweg (Daheim, 1960; Fürstenberg, 1962). Aber es finden sich auch Verweise, daß Menschen aus stabilen Berufstätigkeiten mit überdurchschnittlichen Einkommen von abhängiger Beschäftigung in die Selbständigkeit wechseln, um nicht weisungsgebunden zu sein und eigene Ideen zu verwirklichen, und dabei gelegentlich auch bewußt Einkommenseinbußen in Kauf nehmen.

Um die Verschiedenheit der „Einstiegslogiken“ zu erfassen und zu thematisieren, wurden so aus dem breiten Kontinuum von Rekrutierungskanälen idealtypisch die beiden Pole bestimmt (Bögenhold 1985, S. 243f.; Bögenhold, 1987): Einmal finden sich die Personen, die sich aus einer „Ökonomie der Selbstverwirklichung " selbständig machen und die vergleichsweise gute Arbeitsmarkt- und Einkommenschancen in abhängiger Beschäftigung hatten bzw. hätten. Dem wurde idealtypisch die Gründung gegenübergestellt, die einer „Ökonomie der Not" gehorcht. Hier handelt es sich um Menschen mit fehlenden oder nur geringen Chancen auf eine abhängige Beschäftigung. Sie werden in die „kleine“ Selbständigkeit gedrängt, weil sie eben keine oder kaum Chancen auf dem Arbeitsmarkt haben bzw. sehen. Eine solche idealtypische, zu heuristischen Zwecken getroffene Unterscheidung der Motive für den Schritt in die Selbständigkeit hat insofern einen soziologischen Wert, als sie darauf verweist, daß es mehr als nur eine Eintrittslogik gibt. Aber auf längere Sicht kann diese Unterscheidung nicht vollends befriedigen. Es muß der diesbezüglichen, noch rudimentär entwickelten soziologischen Forschung darum gehen, von einem heuristischen und analytisch-deskriptiven zu einem empirisch vermittelten, stärker theoriegeleiteten Verständnis der divergenten sozioberuflichen Prozesse in das Unternehmertum zu gelangen. Die folgenden Ausführungen stützen sich auf eine empirische Pilotstudie, die diese von mir selber aufgestellte Forschungsforderung ernst genommen hat.

\section{Wege in das Unternehmertum: Empirische Befunde}

Die verfügbaren statistisch quantifizierbaren Angaben über die berufliche Fluktuation in das Unternehmertum, wie sie in der gebotenen Knappheit eingangs dargestellt wurden, geben uns darüber Auskunft, $d a \beta$ in bestimmten Zeiträumen eine bestimmte Zahl von Menschen in die Selbständigkeit wechselt. Der Verfügbarkeit und Dignität der statistischen Daten sind Grenzen gesetzt, etwa wenn es um die Beantwortung weiterer Anschlußfragen (berufliche Herkunft, regionale Verteilung, Alter, Geschlecht etc.) geht. Doch unbeschadet von der Frage nach der ,informativen Tiefe" dieser quantitativen sozial-statistischen Daten wird ein anderer Bereich völlig im Dunkeln belassen: Jenseits des Faktums, $d a \beta$ ein Mobilitätsstrom mit einer bestimmten Geschwindigkeit fließt, wissen wir nicht, wie dieses von den Beteiligten im Einzelfall - also auf individueller Ebene - realisiert wird. Genau das ist die Frage nach dem modus operandi des Selbständigmachens. Für ein adäquates soziologisches Verständnis von Sozialstrukturen und deren Veränderung dürfen wir uns nicht damit begnügen, der Rolle der Akteure und ihrer individuellen Strategien einen Platz zuzuweisen, der bestenfalls noch in den Kulissen der Diskussion angesiedelt ist. Keineswegs möchte ich für eine radikale Umdrehung der Gewichte plädieren, aber für eine Besinnung darauf, daß zwei Untersuchungsperspektiven vonnöten sind, welche jeweils einen Wert für sich haben und vor allem in ihrer Komposition fruchtbar zur holistischen Vermessung sozialer Phänomene beizutragen vermögen. Gefordert sind soziologisch-mikroanalytische Untersuchungen, die nicht gegen makrosoziologische Strukturuntersuchungen ausgespielt werden sollen, sondern geradezu deren basale Grundlagen sind. Konkret: Ziel soll es sein, die Kombination von Motiven und strategischen Konzeptionen in Verbindung mit den jeweiligen Ressourcenallokationen zu ergründen, welche in dem sozialen Prozeß von Firmengründungen eingesetzt werden. Denn der Wechsel von einer abhängigen zu einer selbständigen Erwerbstätigkeit erscheint nur aus der Perspektive der amtlichen Statistik als Akt eines Momentes, als unmittelbarer Wechsel von dieser zu jener Kategorie, aber für die Einzelnen bedeutet es mehr wie ein Griff in den Schrank, um einen anderen Anzug herauszunehmen. Vielmehr stellt dieser Wechsel einen ausgesprochen dynamischen Prozeß dar, der solchermaßen nur in strategischprozessuraler Perspektive aufgefaßt werden kann und der auch mit neuen Konstruktionsprozessen individueller biographischer Entwürfe einhergeht.

Ein sozial- und wirtschaftsstatistisch abgesicherter Befund einer gesellschaftlich objektiven „Erlangbarkeit" ist nicht unmittelbar identisch mit den „Freiheitsgraden“", die die einzelnen Akteure indi- 
viduell zur Wahl ihrer Entscheidungen haben ${ }^{3}$. Die Tatsache, daß Arbeitslosigkeit heute vermehrt Menschen stimuliert, sich beruflich in Richtung einer selbständigen Erwerbsarbeit zu orientieren, bedeutet ja beispielsweise nicht, daß der Weg in die (meistens kleingewerbliche) Selbständigkeit nun auch von jedermann/-frau ohne Schwierigkeiten realisiert werden kann. Industriesoziologische Untersuchungen haben gezeigt, daß beispielsweise auch Arbeiter häufig den Traum von einer eigenen Firma hegen, um ihrer Berufssituation entfliehen zu können, ohne daß sie diesen Traum praktisch umsetzen konnten (Chinoy, 1955; Goldthorpe et al. 1968). Oder bedenken wir, daß wesentlich mehr Arbeitslose gerne beruflich selbständig tätig wären (vgl. die empirischen Befunde bei Brinckmann, 1984), als es tatsächlich probiert haben, so ließe sich vermuten, daß bei den einzelnen Individuen verschieden abgesteckte Aspirationszonen einerseits, aber auch ungleich verteilte „objektive“ Chancen, Risiken und Barrieren vorliegen, die in ihrer jeweiligen Kombination entweder eher fördernd oder aber behindernd wirken. Die Frage lautet, wem die berufliche Passage in das Unternehmertum offensteht, von wem sie beschritten wird und wie die einzelnen Schritte in die Selbständigkeit eingeleitet werden. Denn daß der Weg in die Selbständigkeit schon aus Wettbewerbsgründen und wegen Grenzen der Marktelastizität nicht von allen denen beschritten werden kann, die sich in vergleichbaren Soziallagen gemeinsam wissen, liegt auf der Hand. Strukturell steht der Mobilitätskanal in die Selbständigkeit immer nur einigen offen, so daß gesellschaftlich positiv oder negativ selektiert wird.

Ausgehend von diesem allgemein-theoretischen Problemverständnis wurden in einer "qualitativ" ausgerichteten Pilotstudie, über deren Ergebnisse im weiteren zu berichten ist, etwas mehr als zwei Dutzend Berufswechsel in die selbständige Erwerbsarbeit aufgearbeitet und soziologisch rekonstruiert. Es handelt sich hier um eine Totalerhebung von Karrieren in die Selbständigkeit, die sich im Anschluß an die Stillegung eines Großbetriebes mit zuletzt etwa 2100 Beschäftigten ergeben hatten, und zwar in einem Zeitraum von bis zu zweieinhalb Jahren nach der Schließung Ende des Jahres 1983. Zwar sind die Berufsverläufe dieser „neuen“ Selbständigen für das gesamte Gründungsgeschehen im statistischen Sinne nicht unbedingt repräsentativ, dafür bieten sie aber den beson-

${ }^{3}$ vgl. die grundsätzlichen Ausführungen bei A. Schütz, T. Luckmann, 1975, S. 102f. Ähnliche Argumente finden sich mit Rückgriff auf empirische Studien und in anderer „Aufmachung" auch in dem Skript von K. U. Mayer, 1988. deren methodologischen Vorzug, daß hier Personen in einem Sample versammelt sind, die mit unterschiedlichen Attributen (Alter und Qualifikation) aus demselben Betrieb kommen und zu demselben Zeitpunkt vor dieselbe Situation des „Was nun?" gestellt werden und allesamt mit dem Schritt in die selbständige Erwerbstätigkeit antworteten. ${ }^{4}$

\subsection{Resultate und Motive des Sich- Selbständigmachens}

Die in der vorliegenden Pilotstudie rekonstruierten jeweiligen Wege in die berufliche Selbständigkeit verliefen für die einzelnen insofern bereits unterschiedlich, als sie entweder chronologisch unmittelbar oder erst über andere berufliche Zwischenetappen dorthin führten, als der Prozeß zeitlich schneller oder weniger direkt ablief und als er insgesamt strategisch eher von längerer Hand geplant oder mehr zufällig war. Deshalb schien es forschungslogisch opportun und geboten erst etwa zwei bis zweieinhalb Jahre nach der Schließung des Betriebes (eine Werft) mit der empirischen Untersuchungsphase zu beginnen. Damit gab es eine wesentlich größere Gewähr, daß nun auch alle die, die sich beruflich in die Selbständigkeit bewegen wollten, dort zwischenzeitlich auch wirklich angekommen waren. Aus zwei Gründen erschien das bedeutsam: Einmal ließ sich so bereits bei einer Reihe von „Selbständigenfällen“ auf einen - freilich auch eher knappen - Zeitraum der neuen Tätigkeit in der Berufsrolle der „Nichtabhängigkeit" zurückblicken. Das bot die Chance, die Prozeßhaftigkeit der beruflichen Veränderung soziologisch rekonstruieren zu können und darüber hinaus den Grad des geschäftlichen Erfolges bzw. Mißerfolges bereits ansatzweise zu bilanzieren. Zum anderen war die berufliche Mobilität in die Selbständigkeit verglichen mit den Verbleibquoten und Fluktuationsströmen der gesamten Belegschaft deutlich in der Minderzahl. Damit bestand die Möglichkeit, jedem einzelnen Fall von neuer Selbständigkeit im Anschluß an eine abhängige Beschäftigung in dem stillgelegten Großbetrieb berufsbiographisch auf die Spur zu geben.

Durch die Kombination von verschiedenen Recherchen und vor allem auch durch die intensive Nachfrage bei den bereits „bekannten“ Selbständigen nach den Namen wei-

${ }^{4}$ Die Forschungsgruppe des DFG-Projektes „Folgewirkungen einer Betriebsstillegung - Mobilitätsprozesse auf dem lokalen Arbeitsmarkt" hat mich beim Feldzugang dankenswerterweise unterstützt. Für den weiteren Untersuchungsrahmen vgl. Forschungsgruppe Betriebsstillegung, 1989. 
terer ehemaliger Kollegen, die heute selbständig sind, wurde versucht, eine möglichst vollständige Liste der Selbständigenexistenzen $\mathrm{zu}$ erstellen, die aus der Betriebsstillegung hervorgegangen sind. So konnte deren Zahl ständig komplettiert werden und als Ergebnis ist dann letztlich gewissermaßen eine Totalerhebung herausgekommen, denn die "Gesamtdunkelziffer" (unbekannte Selbständigenfälle und Verweigerer) kann nach einem solchen harten Ringen um jeden Einzelnen als äußerst niedrig angesehen werden ${ }^{5}$. Von der Gesamtzahl der mehr als 2100 ehemaligen Belegschaftsangehörigen konnten 25 Selbständige ermittelt werden, die zum Zeitpunkt Ende 1986/Anfang 1987 wirtschaftlich aktiv tätig waren und über die dezidierte Informationen vorliegen. Mit fast allen wurden in deren Wohnung oder Firma zeitlich ausgiebige detaillierte Interviews geführt ${ }^{6}$.

Die folgenden Ausführungen fußen auf diesen beruflichen Karrieren in die Selbständigkeit, welche transparent gemacht werden konnten und welche auch mit einer gewissen zeitlichen Stabilität in der Selbständigkeit verharrten, wenngleich nicht alle gleichermaßen als erfolgreich zu bezeichnen sind. Ihre wirtschaftlich-unternehmerischen Tätigkeiten werden hier als Firmen bezeichnet, und zwar unabhängig von ihrem tatsächlichen Aussehen und Schwerpunkt, unabhängig ob nun ein Firmenname, Firmengelände oder ein Firmenschild existiert oder vorgesehen ist, d. h. auch eine freiberufliche Übernahme von Schreibarbeiten wird hier beispielsweise als Firma mitgezähit. Die 25 neuen Selbständigen, die aus dem Schoße der Betriebsschließung hervorgegangen sind, entfielen auf 22 Firmen. Drei Firmen waren jeweils Gründungen von Zweier-Teams, in denen ehemalige Kollegen zu Kompagnons geworden waren. Nicht alle dieser 22 Firmen waren Neugründungen, sondern es gab auch Betriebsübernahmen und/oder den Rückzug auf eigene Firmen, die bereits existierten und vorher im Nebenerwerb betrieben wurden (insgesamt fünf Firmen). Der größte Teil der Selbständigkeiten entfiel dabei auf den Bereich, der in der Wirtschaftsstatistik gewöhnlich Restposten-ähnlich als ,.Sonstige Wirtschaftsbereiche (Dienstleistungen)" bezeichnet wird (15 Personen mit 14 Firmen). Fünf weitere gehörten in die Sparte „Handel und Verkehr", die auch zum Tertiären Sektor gehört. Im güterproduzierenden Gewerbe entstanden zwei neue Firmen, die von je zwei Gründern etabliert wurden. Ein Betrieb, der zuvor existierte und bereits im familiären

\footnotetext{
${ }^{5}$ Gerade weil die Verbleibskategorie der Selbständigen zahlenmäßig vergleichsweise gering vertreten ist, haftete den Einzelnen in ihrer Eigenwahrnehmung und in der Wahrnehmung seitens der anderen ehemaligen $\mathrm{Be}$ schäftigten stets ein wenig die Attitüde des Besonderen an (im positiv oder negativ abweichenden Sinne des Exotischen). Die Konsequenz dessen war, daß eigentlich fast alle die Namen zumindest einer Reihe von (anderen) Selbständigen kannten.

"Weitergehende methodische Informationen enthält das Manuskript: Bögenhold, 1988.
}

Nebenerwerb betrieben wurde, war in der Landwirtschaft.

Da die Frage nach den Beschäftigungseffekten von Unternehmensgründungen in der arbeitsmarkttheoretischen Diskussion ein ebenso beliebtes wie umstrittenes Thema ist, sei hier diesbezüglich eine knappe Antwort gegeben, auch weil das Material keine zu verallgemeinernden Schlüsse zuläßt. Vier der Betriebe (darunter drei Neugründungen) schufen dauerhaft neue Arbeitsplätze und wurden selber als Arbeitskraftnachfrager aktiv. Spitzenreiter waren die beiden Betriebe des produzierenden Gewerbes (Metallverarbeitung), die etwa zweieinhalb Jahre nach der Gründung jeweils etwa 20 Personen beschäftigten. Die übrigen Firmen hatten bis dahin keine eigenen nennenswerten Effekte als stetige Nachfrager von betriebsfremden Arbeitskräften gehabt. Vereinzelt wird Teilzeitarbeit nachgefragt wie etwa für Schreibarbeiten. Doch das sind bei den meisten Firmen eher Gelegenheitsjobs, die hin und wieder auftreten. Bei fast allen diesen Firmen läßt sich beobachten, daß auch bei mitunter vergleichsweise langen Arbeitszeiten aus Kostengründen versucht wird, ein möglichst großes Quantum an anfallenden Arbeiten selber abzudecken und zu übernehmen. Wenn die Verrichtung von Tätigkeiten delegiert wird, dann zuallererst unbezahlt an Verwandte und Freunde. Insbesondere sind das Ehepartner, Kinder, Eltern, Geschwister und enge Freundinnen und Freunde der Gründer.

Neben der Frage, was für Firmen gegründet wurden, ist von Interesse, wer die Gründer sind. Das zielt auf die Frage, ob es eine einheitliche, gemeinsame Logik der neuen Firmeninhaber für ihren beruflichen Schritt in die Selbständigkeit gibt oder ob sich gegebenenfalls verschiedene soziale Muster der beruflichen Mobilität in das Unternehmertum finden. Eingeschlossen in die Frage nach dem „Wer?" ist auch die Frage nach den Motiven, nach dem „Warum?“". Da es bei einer kleinen Zahl wie der der hier betrachteten Selbständigen wenig Sinn macht, mit Prozentangaben zu operieren, seien nur ein paar ungefähre Hinweise auf die einzelnen Gruppengrößen gegeben: Das Verhältnis von ehemaligen Arbeitern zu Angestellten unter den ,neuen" Selbständigen ist umgekehrt proportional zum Verhältnis der Werftbelegschaft: Ein Viertel kommt aus der Arbeiterschaft (Facharbeiter und angelernte Arbeiter) und drei Viertel sind ehemalige Angestellte. Wie stand es nun um deren Motive? Hatten sie berufliche Alternativen zu dem Schritt in die Selbständigkeit und war er insofern eher ,freiwillig" oder ,erzwungen“? 
Bei den selbständig-originären Betriebsgründungen im Anschluß an die abhängige Beschäftigung gibt es ein uneinheitliches Bild: Vier hatten mehrfach vergeblich versucht, einen neuen Arbeitsplatz zu finden. Und erst als diese Suche von Mißerfolg gekrönt war, stellte sich der Gedanke bzw. die konkrete Möglichkeit für eine selbständige Erwerbsarbeit ein. Diese Selbständigkeiten lassen sich als „erzwungene“ kennzeichnen, weil sie sich vor dem Hintergrund fehlender Alternativen ergeben haben und weil sie nicht eröffnet worden wären, wenn sich eine entsprechende Beschäftigung in abhängiger Beschäftigung ergeben hätte. Bei einem ehemaligen Arbeiter und heute selbständigem Kurierfahrer, der ein Fahrzeug hat, welches er als Subunternehmer im Nahverkehr einsetzt, lautet es zugespitzt folgendermaßen: „Aus schierer Verzweiflung hab' ich das gemacht. Irgendwas mußte ich ja anfangen. Wie alt war ich? 50 , nee 51". Desweiteren handelte es sich um einen Copyshop, ein Schreibbüro und einen „Erledigungsservice“.

Anders die Situation bei den restlichen: Fünf ehemalige Angestellte wußten sofort, daß sie sich selbständig machen wollten und haben insofern keine Bewerbungen durchgeführt. Man kann davon ausgehen, daß sie mit ihrer Qualifikation sicherlich nicht chancenlos im Hinblick auf neue Beschäftigungsverhältnisse gewesen wären. Fünf weitere Selbständige hatten Alternativen, die sie entweder (a) nicht wahrnehmen wollten, (b) nur vorübergehend mit der Option annahmen, daß es „nur für eine Zeit sein sollte“, oder aber (c) erst nach einer Negativ-Erfahrung bewußt in Richtung Selbständigkeit verließen.

Ex. 1: „Einmal Schiffbau war genug. Ich mach' keine halben Sachen, ich mach' ganze Sachen" (Ingenieur, der zu einer anderen Werft hätte gehen können, und der stattdessen hinterher ein Beratungsbüro eröffnet hat).

Ex. 2: „Ziemlich unmittelbar stand eigentlich fest: Wir machen irgendwas auf eigenen Beinen, wir machen uns selbständig. Nochmal neu irgendwo in 'nem Betrieb anfangen, das wollten wir nicht. Aber was wir nun genau machen wollten, das wußten wir noch nicht so richtig. Wir wußten nur, daß wir irgendwas zusammen machen wollten. ... Ja, und dann kam das Angebot von (...), das wir da anfangen könnten; die wollten uns haben als Spezialisten. Wir waren ja Know-How-Träger. Und da sind wir dann hingegangen. Aber uns war gleich klar, daß wir da nicht lange bleiben wollten, wir wollten was selber machen. Das stand da bereits fest. Und das ganze Klima da bei (..), das hat uns nur bestätigt, das war also ganz anders, wie wir das gewohnt waren, das war nur Drängelei und Drangsalei, gefiel uns gar nicht, und finanziell hatten wir auch weniger als vorher, außerdem dann immer die lange Fahrerei, v. a. im Winter; hat man woanders in Großstädten zwar genauso lange, aber das war nichts. Da haben wir immer gesagt: ,Das Schönste in Bremerhaven ist die Autobahn nach Bremen“" (Ingenieur, der mit einem Kollegen zusammen ein Büro im Bereich Konstruktion und Beratung eröffnet hat).

Ex. 3: „Weil ich nicht gehorchen wollte. Da hab' ich mir gesagt, das hab' ich nicht nötig. Nicht, wenn man mir nur Knüppel zwischen die Beine schmeißt, wo Sie wieder Befehlsempfänger sind, trotz großen Titels“ (Jurist, der auf der Werft zum oberen Management gehörte und danach eine hoch dotierte Tätigkeit an der Spitze der Verwaltung eines privaten Wirtschaftsbetriebes übernahm, aber wegen ständiger Konflikte kündigte und eine kleine Kanzlei eröffnete).

Schließlich gab es auch den Fall, daß bereits im Wissen, sich selbständig machen zu wollen, zuvor noch gezielt eine abhängige Beschäftigung aufgenommen bzw. eingegangen wurde, um sich einen Kundenstamm aufzubauen:

Ex. 4: ,... das ist mir dann egal gewesen, denn ich hatte also im direkten Anschluß zur Schließung noch einen interessanten Job, auch hier im Bremer Raum als Geschäftsführer eines Sportgeschäftes, das mir eigentlich erst die Möglichkeit gegeben hat ..."

Frage: „Aber für Sie war ganz klar, das benutze ich im Grunde als Lernphase und als Warteposition?"“

Antwort: „Ja. Naja, Warteposition gar nicht mal. Es war ja von vornherein auch mit einem bestimmten Ziel ausgerichtet, an diese Leute ranzukommen, die nun alle bei mir in den Ordnern gespeichert sind. Normal wäre es also unheimlich schwierig gewesen, rauszukriegen, wer liefert was. Aus Büchern hätte man sich das anlesen können, aber es ist schon besser, wenn man direkte Kontakte zu den Herstellern, zu den Lieferanten selber geknüpft hat, umso mehr Unterstützung kriegt man dann eigentlich bei der Eröffnung ... Ich kannte dann also die Betriebsergebnisse des Betriebes und konnte das mit meinen vergleichen und wußte gleich in etwa die Verkaufszahlen. Es war also 'n bißchen Spionage mit drin, aber das bleibt nicht aus, weil das letztlich ja allein geschäftliches Interesse war" (Maschinenbauingenieur, der schon seit vielen Jahren irgendwann einmal sein Sporthobby zum Beruf machen wollte und für ein Jahr eine Tätigkeit bei der späteren Konkurrenz als Quasi-Geschäftstraining angenommen hatte).

Die übrigen Selbständigen waren sogenannte „Jein-Fälle": Entweder waren sie zeitlich oder räumlich für bestimmte angebotene Jobs nicht flexibel, wie ein ehemaliger technischer Zeichner, der das Sorgerecht für zwei kleine Kinder hat und deswegen nicht Überstunden oder Schichtarbeit leisten kann. So muß er verschiedene Jobangebote ablehnen und macht dann einen Gemüseladen auf. Oder: Ein Dockmeister bekommt auf mehrere Versuche keine neue Arbeit. In seinem alten Beruf 
als Zimmerer hat man ihm dann eine Arbeit für DM 15,- angeboten: „Doch dafür wollt' ich nicht arbeiten, nachdem was ich auf der Werft verdient hatte". Dann machte er eine kleine Gaststätte in seinem Stadtviertel auf. Ein Patentingenieur bewarb sich mehrfach vergeblich deshalb nur im Raum Bremen, weil er seiner Ehefrau nicht nochmal einen Ortswechsel mit dem damit verbundenen sozialen Neuanfang zumuten wollte. Als er sich dann zur Selbständigkeit fest entschlossen hatte, da bekam er doch noch ein Arbeitsplatzangebot, auf das er nicht mehr einging. Ebenso erging es einem weiteren Ingenieur, der sich lange vergeblich bewirbt und eine Tätigkeit erst angeboten bekommt, als er die Selbständigkeit bereits gedanklich eingeplant hatte: „Später, als ich mich entschlossen hatte, mich selbständig zu machen, da hätte ich 'nen Job bekommen können, aber ich meine, wenn man sich zu etwas entschlossen hat, dann sollte man nicht umfallen, dann bleibt man dabei normalerweise“.

Da im vorliegenden Rahmen eher die harten Konturen der Darstellung von Belang sind und dazwischen nicht mit zu feinem Pinsel gearbeitet werden soll $^{7}$, sei hier zusammenfassend zunächst so viel festgehalten: Wir finden hier gleichermaßen die aus "Not" in die Selbständigkeit Gedrängten, denen für eine weitere Teilnahme am Erwerbsleben keine andere Möglichkeit als der Schritt in die Selbständigkeit bleibt, und die, die sich eher aus einer „Ökonomie der Selbstverwirklichung“ leiten lassen und durchaus Alternativen für andere $\mathrm{Be}$ schäftigungen hatten. Aber es zeigen sich auch verschiedene Mischformen, dernach die Handlungsmuster sich nicht nur solchermaßen exklusiv dichotomisieren lassen, sondern stärker auf eine "sowohl-als-auch-Logik“ hinauslaufen, auf ein eher indifferentes Muster kontingenter Zufälligkeiten.

\subsection{Der modus operandi des Sich- Selbständigmachens}

Die Frage nach dem modus operandi zielt auf den spezifischen Einsatz von Instrumentarien, die im Prozeß des Sich-Selbständigmachens als Ressour-

'Die Fälle der (hauptberuflichen) Übernahmen von eigenen Firmen, die bereits existierten und zuvor nebenberuflich betrieben wurden, bleiben hier unberücksichtigt, weil der Raum zur Darstellung fehlt. Sie sperren sich indes nicht gegen die vorliegende argumentative Beweisführung, sondern fügen sich in diese nahtlos ein. Ausführlicheres vgl. Bögenhold, 1988. cen in unterschiedlichem Maße mobilisiert werden konnten: Geld, Wissen, Sozialkontakte. In fast 130 Interviews mit ehemaligen Beschäftigten des stillgelegten Großbetriebes (vgl. weitergehend Forschungsgruppe Betriebsstillegung 1989), welche nicht selbständig waren, wurde explizit danach gefragt, ob sie daran gedacht gehabt hätten, sich selbständig zu machen. Knapp die Häfte von ihnen hat das strikt verneint, während die anderen „schon mal“ daran gedacht hatten, das dann aber entweder schneller oder langsamer verworfen oder aber auch ernster erwogen und letztlich aus anderen Gründen nicht realisiert hatten. Angst, das Fehlen von Geld, Zeugnissen (Meisterbriefe etc.) oder einer entsprechenden Geschäftsidee hielt diejenigen letztlich $\mathrm{ab}$, die nicht prinzipiell ablehnend gegenüber der selbständigen Erwerbsarbeit waren. Oft findet sich das Antwortmuster, daß zwar schon mal ,spielerisch“ daran gedacht worden war, aber dann diesbezüglich keine weiteren strategischen Schritte eingeleitet wurden, weil die Einzelnen nach gründlicher Abwägung der Vor- und Nachteile und der persönlichen Voraussetzungen und Risiken Bedenken im Hinblick auf eine erfolgreiche Realisierung hatten.

Damit ergibt sich die Frage, ob diejenigen, die sich nun tatsächlich selbständig gemacht haben, bestimmte Voraussetzungen, Chancen und sonstige (positive oder negative) Konditionen hatten, die ihre Entwicklung begünstigten. Mit Blick auf die Tätigkeit, die zum Mittelpunkt der selbständigen Produktion von Gütern und Leistungen gemacht wurde, läßt sich sagen, daß ungefähr die Hälfte der Gründer das als Arbeitsqualifikation aufgeboten bzw. in das neue Geschäft eingebracht haben, was sie in etwa auch vorher in abhängiger Beschäftigung machten oder was zumindest ihrer Ausbildung entsprechend war. Besonders evident ist das beispielsweise bei einer Reihe von Ingenieurbüros, die in ihrer Leistungspalette einen Großteil dessen anbieten, was Gegenstand ihrer vorherigen Tätigkeit auf der Werft war.

Demgegenüber hat sich eine vergleichbar große Zahl nicht unmittelbar auf eine Tätigkeit gestützt, die als adäquat zur Berufsausbildung oder -tätigkeit anzusehen ist: Das sind beispielsweise diejenigen, die eine Gaststätte, einen Gemüseladen, KFZ-Handel, Copyshop, eine Surfschule oder einen Kurierfahrerbetrieb eröffneten. Hier liegen niedrige Barrieren im Hinblick auf die Erfordernisse von (formalen) Qualifikationen vor ( $\mathrm{z} . \mathrm{B}$. (nur) Gesundheitszeugnis oder Führerschein Klasse III) und/oder das Know-How wurde nebenbe- 
ruflich erworben - sei es im Rahmen eines Hobbys wie Surfen, sei es durch Aushilfstätigkeiten bei einem Gemüsehändler auf dem Großmarkt oder durch ein gezieltes (informelles) Praktikum bei einem Verwandten, der ein solches Geschäft (Copyshop) in einer anderen Region hat. Hier handelte es sich also summa summarum entweder um „Jedermanns(-fraus)-Qualifikationen“ oder um Qualifikationen, für die spezifische Interessen prädestinieren und die später ,on the job" eingeschliffen wurden.

Die finanziellen Aufwendungen für die Gründungen waren von Fall zu Fall unterschiedlich hoch, so $\mathrm{da} ß$ sich keine allgemeingültige Auskunft geben läßt. Höchstens bei einem halben Dutzend von Fällen waren Investitionen nötig, die über einhunderttausend Mark lagen - teilweise dann auch beträchtlich darüber. Diese Beträge wurden aus Sparguthaben und über Bankkredite aufgebracht, für die beispielsweise Hypotheken auf Eigenheime aufgenommen wurden. In zwei oder drei Fällen hatten hier außerdem positive Stellungnahmen einer Wirtschaftsförderungsgesellschaft Hilfestellung für die Gewährung von Krediten geleistet. Staatliche Zuschüsse aus sogenannten Existenzgründungsprogrammen spielten durchgehend keine Rolle.

Bei den meisten anderen neuen Selbständigkeiten lag die Höhe des Startkapitals deutlich niedriger. Es waren nicht selten weniger als $10000 \mathrm{DM}$. Diese Gelder wurden aus Erspartem und/oder der Abfindung aufgebracht. Oft war zudem noch ein zweites Haushaltseinkommen durch einen Ehepartner vorhanden. Außerdem konnte die Tätigkeit häufig kostenneutral in vorhandenen Räumlichkeiten begonnen werden. Zum Beispiel: Der Großvater hatte noch einen Schuppen, der erstmal zur Werkstatt taugte; in dem eigenen leerstehenden Laden ließ sich eine Gaststätte einrıchten oder die Büros fanden Platz auf einem noch auszubauenden Dachboden im eigenen Hause. Die meisten hatten solche sachlich-räumlichen Ressourcen, auf die sie zurückgreifen konnten. Der Hauptfinanzierungsengpa $\beta$ bestand häufig eher in der ersten Anlaufzeit. So nutzten fast alle ihre erste Arbeitslosigkeit zur Renovierung und Einrichtung der Räumlichkeiten und zum Anbahnen von ersten Geschäften. Da hier das Einkommen aus der neuen Selbständigkeit mitunter noch sehr spärlich war, griffen fast alle noch auf die Arbeitslosengelder zurück, die vom Arbeitsamt gezahlt wurden, sofern es von der Firmengründung nichts wußte. In diesen Fällen wurde so die erste Experimentier- und Aufbauphase mancher Selbständigkeiten mitunterstützt, auch weil die Einzelnen moralisch im Glauben waren, daß ihnen diese Gelder zustehen, da sie über lange Zeit einbezahlt worden waren.

Die besten Firmenräumlichkeiten taugen für sich selber indes noch nicht zu erfolgreichen Geschäften auf eigene Rechnung. Vielmehr muß es ebenso gelingen, Kunden, Klienten oder Patienten, d. h. Abnehmer von Gütern bzw. Leistungen zu haben bzw. zu gewinnen. Diesbezüglich kommt der Existenz von jeweils relevanten sozialen Kontakten zumindest für die Anfänge der Geschäftstätigkeit eine wesentliche Rolle zu. Das Vorhandensein von sozialen Netzwerken ist zwar auch gewissermaßen beschaffungsseitig bei der Einrichtung der Produktionsstätte wichtig, etwa wenn es darum geht, wo wer welche Maschinen, Fahrzeuge, Büromöbel, Farben usw. hat, die gebraucht und/oder kostengünstig übernommen werden können. Noch wichtiger aber scheint es zu sein, wenn es gewissermaBen absatzseitig um die Vermittlung von Kunden geht. So zeigt sich, daß viele auf ein soziales Netzwerk in einer bestimmten regionalen Verdichtung zurückgreifen konnten, welches unabdingbar ist, um „rein-“ und „ranzukommen“. Der Funktionsmechanismus dieser sozialen Netzwerke, um hier bei dem inzwischen etablierten Begriff zu bleiben, der gewöhnlich J. A. Barnes (1954) als Schöpfung gutgeschrieben wird, aber dessen differenzierte Bearbeitung sich bereits bei G. Simmel (1968, S. $305 \mathrm{ff}$.) unter dem Titel der „Kreuzung sozialer Kreise" findet, drückt sich in den Interviews exemplarisch folgendermaßen aus:

Ex. 1: „Wie sind Sie denn an solche Aufträge rangekommen?"

„Damals war das so, wenn wir so die Kette mal so sehen, Sie kennen einen, und der kennt wieder einen".

„Und das waren alles Leute von der Werft dann?"

„Die ersten ja. So ein, zwei Leute, die ham uns den Weg zu bestimmten Firmen geöffnet, und dann kam das denn da, und jetzt kommen mit der Post schon immer automatisch schon die Anfragen, ehrlich, von Frankreich, unwahrscheinlich. Und wir sind jetzt so, woll'n mal so sagen, daß wir ein gut florierendes Unternehmen sind“.

Ex. 2: „Ich sagte ja eingangs mit dem Schneeballeffekt. Leider war der Schnee 'n bißchen mickrig, daß der Schneeball nicht so schnell ging, aber im Prinzip geht das so: Also, man kennt ja irgendwie Leute, Kollegen von anderen Firmen und da ruft man an. Also Leute jetzt, die vielleicht einem 'nen Tip geben können, so ist das. Und dann fragt man, wie ist das, habt ihr Druck oder so, also ich hab' mich selbständig gemacht, sagt Bescheid, wenn ihr Druck habt, ich helfe gern mit und so, also was man sozusagen als Klinkenputzen bezeichnet. Also man geht rum und sagt das Bekannten, mit denen man irgendwie 
zusammengearbeitet hat oder mal kennengelernt hat, hier ist was und ich mach das sehr günstig und so, ich fang ja erst an und so also kommt das dann ins Rollen. ... Also ich mach' ja nicht irgendwie Arbeiten, die, sagen wir mal, jeder machen könnte: also 'nen technischer Zeichner oder 'n Konstrukteur, verstehn Sie. Dann würde ich ja nichts verdienen. Die Leute wissen ja ganz genau, was was kostet, verstehn Sie, also ich muß schon Arbeiten nehmen, die meiner Qualifikation entsprechen, weil ich dann mehr verdienen kann, aber wenn man nichts hat, wie heißt das? Dann frißt der Wolf auch Mäuse, das ist klar ..."

Ex. 3: „Und Sie hatten vorher keine Vorstellung so über mögliche Kunden, also nur die Idee, wenn ich da und da mal Reklame mache, wird da vielleicht was zurückkommen?"

„Ja, was heißt Idee. Sie kennen jemand, Sie haben zum Beispiel mit Leuten zu tun. Sie haben studiert oder aus ' $m$ Sportverein oder weiß ich wie, ja und dann, betteln ist nicht jedermanns Sache, auch nicht meine, ja aber anrufen und sagen: du hör' mal, habt ihr Probleme oder Bedarf oder weiß ich was, also so fängt es an, und neulich rief mich ein Studienkollege vom TÜV an in Hamburg und sagte zu mir, Mensch, wenn ich das geahnt hätte, daß du dich selbständig gemacht hast, du hättest soundsoviele Aufträge haben können, was haben wir an Geld da an die Firma in Frankfurt bezahlt für Gutachten, und da werden so Berechnungen gemacht, statische Berechnungen, so nach 'ner bestimmten Methode, und da sagt er, du warst ja schon immer pingelig und so weiter, das hättest du gut machen können, er nannte mir also, was da für Aufträge vergeben wurden, es heißt ja jetzt nicht, daß der mir 'nen Gefallen tun kann, das kann er nicht. Erstens entscheidet er das gar nicht, aber wenn er sagt: Ich kenn' da noch einen, also jetzt zufällig, setzt Euch mit dem in Verbindung, verstehn Sie, dann ist er außen vor. Dann hat er also, mein Studienkollege, damit nichts zu tun, ich muß mich mit den, weiß ich was, Einkäufern und so weiter auseinandersetzen, aber Sie haben da 'n Nummernschild dann ...".

Offener oder verdeckter findet sich in fast allen Fällen ein soziales Geflecht von Reziprozitätsstrukturen, dessen sich die einzelnen bedienen oder zu bedienen trachten. Statt eines solchen eher passiven Rückgriffs findet sich gelegentlich darüber hinaus auch die proaktive Strategie, bestimmte soziale Bedingungen erst zu schaffen, beispielsweise wenn Personen bewußt in bestimmte Verbände gehen und dort Ämter übernehmen, weil sie wissen, daß dort die „signifikanten Anderen“ (A. Schütz) im Hinblick auf das eigene Geschäft anzutreffen sind. Wohlkalkulierte und wohlbegründete Strategien finden sich ähnlich im Bereich der Wartung und des Ausbaus des Kundenstamms.

Ex. 4: „Ja, zuerst da hab’ ich mich anders verhalten. Da hab' ich gerechnet und geknausert und bin bis sonstwo hingefahren, um möglichst billig einzukaufen, nur die niedrigsten Preise hab' ich akzeptiert. Das hab' ich aber ganz schnell dann seinlassen, weil das 'ne falsche Rechnung ist. Heute kaufe ich nur bei Leuten und Firmen hier im Ort und umzu ein, auch wenn das mal 'ne Mark teurer ist. Aber dafür kennen die mich dann, und wenn die sich nun mal 'nen Auto kaufen, dann müssen die zu mir kommen, weil sie sich verpflichtet fühlen. So läuft das, das funktioniert so also viel besser - meistens jedenfalls. Geht ja nicht, daß ich nur bei denen kaufe und die nicht bei mir".

Ähnlich zielen Kalküle der Imagepflege auf die Etablierung von Kundentreue, etwa wenn der Gastwirt für seine „Rentner vom Großeinkauf auf dem Schlachthof immer so 10 bis 15 Tragetaschen voll Schweineköpfe“" mitbringt. „Da machen die dann Sülze von. Kostet mich ja nichts“. Dieses betriebswirtschaftlich funktionale Verhalten ist nur im Grenzfall Ausdruck naiver Nettigkeit. Ein Surfschulbetreiber begründet beispielsweise die eingeräumte Möglichkeit, daß die Schüler seine Werkstätten benutzen und dort selber reparieren können, als bessere Serviceleistung und höhere betriebliche Flexibilität:

„Unsere Angebotspalette ist größer als bei anderen. Wir - ich sag ,wir', Sie werden von mir nie hören ,ich', sondern ,wir', das ist eben der (Firmenname) - haben eigene Werkstätten. Die können bei uns kommen und gehen. Können auch selber reparieren, ja. Das entlastet mich ja und ist für die Leute interessant".

Um die sozialen Kontakte zu knüpfen und um sich ihrer erfolgreich $\mathrm{zu}$ bedienen, bedarf es sicherlich im Einzelfall sozialer Talente, die nicht allen Menschen gleichermaßen gegeben sind. Diese „social skills" (Blau 1956) sind im vorliegenden Zusammenhang viel eher ,funktional“ als „extrafunktional", um hier die Dahrendorf'sche (1956) Terminologie aufzugreifen. Alle Faktoren im Zusammenspiel sind in bestimmten Konstellationen notwendig, um erfolgreich eine Firma zu gründen. Doch beileibe nicht alle der hier etablierten Firmen sind schließlich als Musterbeispiele für die Frage: „Wie gründe ich erfolgreich ein Unternehmen?" anzusehen. Es hat sich als außerordentlich schwierig erwiesen, bei Selbständigen im Gegensatz zu ihrem letzten Einkommen auf der Werft präzise Auskünfte über ihre derzeitige Einkommensituation zu erhalten. In vielen Fällen konnte aber über beharrliches Nachfragen (nach dem Motto: besser oder schlechter, viel mehr oder viel weniger, das Doppelte oder die Hälfte?) Klarheit über die Richtung der Einkommensentwicklung erzielt werden. Demnach läßt sich hinreichend gesichert festhalten, daß von diesen neuen Selbständigen die Hälfte ihr Einkommen gehalten oder 
sogar deutlich erhöht hat. Die andere Hälfte teilt sich zu gleichen Teilen in ,Verlierer" und „Indifferente“. Die „Verlierer“, die entweder den Gürtel enger schnallen mußten und auf finanziell reduziertem Niveau mit immensem Arbeitseinsatz in der Selbständigkeit verharrten oder die kurz vor der endgültigen Betriebsaufgabe standen und sich bereits auf die Institution der Sozialhilfe verwiesen sahen, weinten der früheren Tätigkeit manche Träne nach. Das letzte Anteilsviertel der „Indifferenten" sperrt sich gegen die Verortung in einer solchen Gewinner-Verlierer-Taxonomie, beispielsweise weil deren Firma noch zu jung war, um hinreichend gesicherte Einkünfte verzeichnen zu können, was von ihren Betreibern auch nicht anders erwartet worden war.

Die Perzeption und Akzeptanz der neuen Berufsrolle „Unternehmer“ unterscheidet sich längs der Achse des individuellen geschäftlichen Erfolges. Von Mischtönen und sich überschneidenden Schattierungen abgesehen finden sich zwei unterschiedliche Deutungsextrema, die den Modi der bei Goffman (1972, S. 149) beschriebenen „Erfolgs-Story's“ einerseits und „traurigen Geschichten" (sad tales) andererseits entsprechen. In den „Erfolg-Story's“ fungiert der Einzelne als eigener, autonomer Produzent seines Erfolges im Sinne einer selbstgemachten „Wer will, der kann“-Erfahrung mit dem demonstrativen Gestus des „Seht her, was ich geschaffen habe!":

Ex. 1: „Nach meiner Lehre habe ich also in einem Betrieb gearbeitet als Maschinenschlosser, um einfach noch'n bißchen mehr Praxis zu sammeln. Ich habe einige Male die Firmen gewechselt, um in die gesamten Organisationen ' $n$ bißchen mehr reinschnuppern zu können. Warum läuft dies so und hier und da, und hab'mir also überall ein entsprechendes Wissen angeeignet und hab' das natürlich ausgebaut durch irgendwelche Seminare, Kurse, Volkshochschule und was weiß ich alles. Und da war bei mir schon der Gedanke, daß ich mir persönlich eigentlich zu schade bin, für die anderen zu arbeiten, wo es doch in meiner Macht steht, mich selbständig zu machen. ... Und heute: Man ist viel freier, man ist sein eigener Herr, das fängt schon damit an, daß sie jemandem, hier (übergibt seine Visitenkarte, Anm. D. B.) ihre Karte geben können. Wissen Sie, das ist einfach das Gefühl, das kann man auch nicht in Geld messen, ich brauch auch nicht unbedingt 'nen 350 SEL vor der Tür, da gibt es andere Dinge, mit denen ich mir's beweisen kann. Also, wieder zurück in die Abhängigkeit, niemals, das würde ich nie mehr machen, höchstens so als Berater, ja, aber nicht wieder direkt weisungsgebunden im Arbeitsprozeß, wie damals als Angestellter, das würd' ich nicht mehr machen".

Gegenüber einem solchen „Wo ein Wille, ist auch ein Weg" stehen exemplarisch zwei prägnante und umso kürzere Zitate von beruflichen Negativ-Episoden in der Selbständigkeit:

Ex. 2: „Das ist eigentlich ganz schwierig. Ich würde an sich da jeden schon von abraten: Selbständig, das ist nicht so, da fliegen einem keine gebratenen Tauben in 'nen Mund. Das ist: Sie stehen vor einem Nichts".

Ex. 3: „Sie können doch strampeln und tun was Sie wollen, es bringt nichts. Und wie gesagt, entweder, ich hab' zu meiner Frau gesagt, entweder man wird zum Alkoholiker oder man hängt sich auf“"

Die Mobilität in die Selbständigkeit ist für die einzelnen Akteure sehr viel „offener" im Hinblick auf deren Erfolg oder Mißerfolg. Die der Gründung einer Firma sich anschließende ,soziale Flugbahn" (Bourdieu, 1970) ist verglichen mit den beruflichen Laufbahnmustern in abhängiger $\mathrm{Be}$ schäftigung weniger vorstrukturiert und weniger prognostizierbar. Die „Logiken“ dieser relativ offenen Selbständigenbiographien im Hinblick auf Scheitern oder Glücken scheinen (in der idealtypischen Zuspitzung) einfach strukturiert zu sein: Im Fall des Scheiterns sind die einzelnen aus Imperativen der Identitätsbehauptụng in der Eigenperzeption und dem nach außen gewendeten Rollenmarketing nicht primär für ihre beruflichen Schritte selber verantwortlich, während umgekehrt der (relative) geschäftliche Erfolg als persönlicher Erfolg, d. h. als Ergebnis persönlicher Anstrengung und Qualitäten, verbucht wird und, teils auch nur mit der Attitüde des bescheidenen Understatements, ein Bild kreiert wird, demnach der Einzelne als der Dirigent seines eigenen Lebens erscheint, der alle Fäden in der Hand hält.

\section{Soziale Netzwerke und Firmengründungen}

Das vorstehend aufbereitete Material der Rekonstruktion von Firmengründungen enthält bei aller Gedrungenheit der Ausführungen eine Reihe von Ergebnissen, die über ihren unmittelbaren soziolokalen Gewinnungszusammenhang hinausreichen und insofern auch allgemein soziologisch notabel sind. Deshalb werden hier einige Ergebnisse aufgenommen, soziologisch zugespitzt und generalisiert. Entgegen den Annahmen einer Reihe der frühen bekannten Soziologen über die fehlende soziale Mobilität in die Selbständigkeit und die relative Geschlossenheit der Grenze zwischen abhängiger und selbständiger Erwerbstätigkeit verdeutlichen die Ergebnisse verschiedener Studien und statistischer Quellen, daß hier zwar keine Hauptverkehrsstraße gesellschaftlicher Mobilität besteht, aber immerhin doch eine passabel fre- 
quentierte Nebenverkehrsstrecke vorliegt. Der zentrale Ausgangspunkt dieses Beitrages war, daß es kaum Informationen darüber gibt, unter welchen Umständen, mit welchen Zielen und unter Mobilisierung welcher Instrumente einzelne Menschen Unternehmer werden. Jenseits des Faktums also, daß ein Mobilitätsstrom mit einer bestimmten Geschwindigkeit fließt, wußten wir bisher nicht, wie eine solche berufliche Passage in das Unternehmertum von den Beteiligten im Einzelfall realisiert wird.

Zwar können die in dem Untersuchungssample analysierten Fälle im statistisch-quantitativen Sinne nicht unbedingt Repräsentativität für sich beanspruchen, weil sie sich etwa nach Branche und Region einerseits und darüber hinaus nach Alter, Geschlecht und Qualifikation der Akteure vom bundesdeutschen Durchschnitt unterscheiden, und doch läßt sich dessen ungeachtet in dem Besonderen des Designs und der Ergebnisse dieser Pilotstudie auch das soziologisch Allgemeine finden ${ }^{8}$. Es geht hier also mit anderen Worten um den $\mathrm{zu}$ extrahierenden soziologischen „Strukturkern“.
Um zu betonen, daß es nicht nur eine umfassende soziale „Logik" für den Weg in eine selbständige Erwerbstätigkeit gibt, sondern daß differente Logiken der Mobilität existieren, wurden idealtypisch zu heuristischen Zwecken die beiden Pole des breiten Kontinuums von Rekrutierungskanälen und -mustern bestimmt, nämlich einerseits die Mobilität, die ihre Rationalität in einer „Ökonomie der Not" hat, und andererseits die, die einer „Ökonomie der Selbstverwirklichung “ folgt. Die geforderte empirische Überprüfung und Konkretisierung (vgl. Bögenhold, 1987a, S. 33) zeigt, daß beide Extrema gleichermaßen vertreten sind, und zwar sowohl die aus "Not" in die Selbständigkeit Gedrängten, denen für eine weitere Teilnahme am Erwerbsleben keine andere Möglichkeit als der Schritt in die Selbständigkeit bleibt und die keineswegs vorrangig von sogenannten Selbstverwirklichungswerten beseelt sind, als andererseits auch die, die sich eher von einer Rationalität und Faszination der Selbstbestimmung leiten lassen und durchaus Alternativen für andere (abhängige) Beschäftigungsverhältnisse hatten. Aber es zeigen
Arbeitsmarktlage:

berufliche Alternativen in abhängiger Beschäftigung

\begin{tabular}{c|ccc|}
\multicolumn{1}{c}{ schlecht } & gering & indifferent & hoch \\
\cline { 2 - 4 } indifferent & I & II & III \\
gut & IV & V & VI \\
& VII & VIII & IX \\
\hline
\end{tabular}

Diagramm 1 Verschiedene Ausgangslagen für den Weg in die Selbständigkeit.

${ }^{8}$ In fast allen Studien der empirischen Sozialforschung, die mit sogenannten qualitativen Verfahren operieren, setzt die Forschung - man kann fast sagen ,typischerweise" - am Besonderen an, um daraus das Allgemeine (im Sinne des „Strukturkerns") extrahieren zu wollen. Auch hierin ist desübrigen die epochale Studie über die "Arbeitslosen von Marienthal" (M. Jahoda et al., 1969) eine prominente Vertreterin: Schließlich spiegelten Marienthal und seine dortigen Verhältnisse mitnichten die Arbeitsmarktsituation im eben statistisch-repräsentativen Querschnitt (weder Österreichs noch darüber hinaus) wider. Dennoch konnten sie mit der Untersuchung am Fall stellvertretend für das "soziale Ganze“ unterschiedliche Rationalitäten im Umgang mit dem Phänomen Arbeitslosigkeit eruieren. sich hier in concreto auch verschiedene Mischformen, soziologisch auch deshalb interessant, weil diese letzteren Karrieren der Zahl nach dominieren. Demgemäß lassen sich die Handlungsmuster nicht nur in zwei „Logiken“ exklusiv dichotomisieren, sondern laufen stärker auf eine ,sowohl-alsauch-Logik" hinaus, auf ein eher indifferentes $\mathrm{Mu}$ ster kontingenter Zufälligkeiten. Bezogen auf das folgende Kreuzschema heißt es, daß eben nicht die beiden „Typen“ II und IX dominieren, sondern daß die dazwischen liegenden „Typen“ ebenfalls deutlich vertreten sind. Besonders die „Typen“ V, VI, aber auch II und III sind zu berücksichtigen (VII und IV sind dagegen eher unwahrscheinlich). 


\begin{tabular}{|c|c|c|}
\hline & & asiert \\
\hline & $\begin{array}{l}\text { primär auf sachlichen } \\
\text { Produktionsmittein }\end{array}$ & $\begin{array}{l}\text { nicht primär auf sachlichen } \\
\text { Produktionsmitteln }\end{array}$ \\
\hline gut & I & II \\
\hline schlecht & III & IV \\
\hline
\end{tabular}

Diagramm 2 Verschiedene Produktionsfelder von Firmen.

Bei der Frage nach den Instrumentarien, die für den Schritt in die Selbständigkeit realisiert werden (müssen), zeigt sich, daß die Ressourcen Geld, Wissen und Sozialkontakte in jeweils unterschiedlicher Gewichtung ineinanderspielen. Die eingangs behandelten Positionen der Klassiker mit ihren pessimistischen Einschätzungen der beruflichen Mobilität in die Selbständigkeit dürften auch darauf zurückzuführen sein, daß sie wohl nur an solche Firmentätigkeiten gedacht hatten, für deren Etablierung immense Finanzmittel erforderlich sind. Weite Teile der selbständigen Produktion von Dienstleistungen basieren indes nicht primär auf der Existenz von sachlichen Produktionsmitteln mit nennenswertem Kapitalvolumen. In einem Vier-Feld-Schema lassen sich idealtypisch verschiedene Produktionsbereiche und -besonderheiten zeigen, die unterschiedliche sozioökonomische Lagen für die Firmenbetreiber indizieren.

Ebensowenig wie es nur eine soziale Logik der Mobilität in das Unternehmertum gibt, ebensowenig findet sich das Unternehmertum im Sinne eines homogenen Feldes mit nur einer Produktionscharakteristik. Zumindest ist zwischen einer Güterund Dienstleistungsproduktion zu differenzieren. Bei der Dienstleistungsproduktion tritt die Bedeutung von sachlichen Produktionsmitteln in den Hintergrund, während diese in der Güterproduktion nicht immer, aber gewöhnlich im Vordergrund stehen, was natürlich das Erfordernis von „Wissen“ nicht ersetzt. Ob die Marktposition nun "gut" oder „schlecht" ist, hängt von einer Reihe von unterschiedlichen Faktoren ab, von denen einer der Grad der gesellschaftlichen Verfügbarkeit und Erlangbarkeit bzw. der relativen Monopolisierbarkeit der einzelnen Produktionsfaktoren ist: Je üppiger bestimmte Instrumentarien in der Gesellschaft vorhanden sind, desto leichter sind sie individuell zu substituieren. Demzufolge sind dann die Einstiegsbarrieren niedriger und desto schlechter ist letztlich (idealtypisch) die Marktlage. Die
Felder "II“ und "IV“" unterscheiden sich so beispielsweise in der Form des notwendigerweise eingebrachten Wissens: individuell angeeignetes Spezialwissen, das häufig durch staatliche Reglementierungen (Ausbildungsverordnungen etc.) außerdem künstlich knapp gehalten wird, versus Jedermanns-/fraus-Wissen bzw. schnell und einfach erwerbbare Kenntnisse und Fähigkeiten. $\mathrm{Ob}$ die Marktlage nun für die einzelnen Firmen „gut“ oder „schlecht“" ist, ist nie ausschließlich von den objektiven Charakteristika der jeweiligen Branchen abhängig, sondern immer auch Ergebnis und Ausdruck von verschieden erfolgreichen Strategien der Kundensuche und -sicherung. Und diese Strategien sind unmittelbar verknüpft mit der Existenz von spezifischen sozialen Netzwerken. ${ }^{9}$

${ }^{9}$ Hinzu kommt ein thematischer Aspekt, der hier aus Gründen des fehlenden Platzes weitgehend unberührt bleiben mußte und der letztlich auch empirisch gründlicher $\mathrm{zu}$ erforschen ist. Gleichwohl enthält dieser Aspekt eine eminent wichtige Forschungsperspektive, welche es detaillierter zu explizieren und theoretisch zu untermauern gilt, nämlich die nach verschiedenen psychosozialen Dispositionen der einzelnen Akteure. Von der Vorstellung ausgehend, daß die psychischen Infrastrukturen und Haushalte der Individuen, und zwar auch bei solchen in „objektiv“" gleichen Lagen, ungleich ausgestattet sind, wäre in einer differentiellen soziologischen Aktionsforschung zu fragen, inwieweit sich hier generell auch entsprechende Perzeptions-, Aktionsund Reaktionsmuster empirisch und theoretisch eruieren lassen. Diese Perspektive drückt sich sinnfällig in der Parabel von den zwei Fröschen aus, die auf dem Rand einer Milchkanne sitzen und in diese dann hineinfallen: Der eine Frosch resigniert und ertrinkt, während der andere nicht aufgibt und unermüdlich in der Milch weiterschwimmt, bis er mit einem Mal auf einem Butterklumpen fußfassen und hinausklettern kann. Einige unsystematische und eher reservierte Überlegungen bezüglich einer Selbständigenforschung, die sich auch um das „Psychogramm“ der Akteure kümmert, finden sich bei Bögenhold, 1985, S. $243 \mathrm{ff}$. 
Auf die Bedeutung von sozialen Netzwerken als Schlüsselfunktion im ProzeB der Arbeitsplatzsuche, hatte in den 70er Jahren M. S. Granovetter $(1973,1974)$ hingewiesen. Er kam in seiner Untersuchung zu dem Schluß, daß ein Mangel an Informationen bzw. ein schlechter Zugang zu Informationen für Menschen auf dem Arbeitsmarkt nachteilig ist. Menschen mit diversifizierten Sozialkontakten, die anstelle nur weniger intensiver, und d.h. selbstreferentieller, viele schwächere Bekanntschaften und Kontakte (weak ties) haben, haben einen besseren Zugang zu Informationen und damit zu Möglichkeiten, neue oder bessere Arbeitsplätze zu finden ${ }^{10}$. Die hier präsentierten Ergebnisse lesen sich wie eine "Übersetzung" des Themas der Jobsuche auf den sozialen Prozeß von Firmengründungen. Die Existenz solcher sozialen Netzwerke spielt bei einer erfolgreichen Firmengründung eine zentrale Rolle. Dabei kommt der Existenz von mobilisierbaren Sozialkontakten auf dem Weg in das Unternehmertum sowohl beschaffungs- wie absatzseitig ein hoher Stellenwert zu. Um Informationen, Hilfe, Kontakte und Kunden zu bekommen, bedarf es eines vielfältigen StrauBes sozialer Beteiligungsformen. Man muß das entsprechende soziale Terrain wie seine Westentasche kennen, will die Firma gegründet und am Leben erhalten werden ${ }^{11}$.

Bei der Frage nach der Komposition der Ressourcen, die den Schritt in die Selbständigkeit realisieren helfen, gilt es - summa summarum - festzuhalten, daß es offenbar nicht nur auf eine einzige Ressource (wie etwa Geld) ankommt, sondern daß grundsätzlich eine entsprechend abgestimmte $\mathrm{Zu}$ sammensetzung von verschiedenen Ressourcen essentiell zu sein scheint. Bei aller Wichtigkeit der Ressourcen Geld und fachliches Know How, die im Einzelfall in ihrem Zusammenspiel unterschiedlich akzentuiert sind, darf die Bedeutung von sozialen Netzwerken keineswegs unterschätzt werden. Sie sind vielmehr als eine Schlüsselvariable

${ }^{10}$ In der Bundesrepublik hat es in den 80er Jahren eine Reihe von Arbeitsmarktstudien gegeben, die (auch) auf die informellen Suchkanäle rekurrierten (vgl. Windolf/Hohn, 1984, vgl. außerdem die Ausarbeitungen und Überblicke bei Blaschke, 1987, Deeke et al. (Hrsg.), 1987, Preißendörfer/Voss, 1988).

"Die ersten Ergebnisse des (mit quantitativen Verfahren arbeitenden) soziologischen Forschungsprojektes über „High-Tech-Gründer" von H. Aldrich und Kollegen bewegen sich auf derselben Forschungsfährte und deuten ebenfalls auf die Relevanz der sozialen Netzwerke hin. Vgl. z. B. H. Aldrich et al. 1987. anzusehen. Die Berufspassage in das Unternehmertum erscheint als ein ausgesprochen dynamischer Prozeß, der in vielfältige soziale Bezüge eingebettet ist. Die Existenz und der Einbezug von sozialen Netzwerken erklärt nicht „alles“, aber umgekehrt können wir ohne deren Berücksichtigung die Etablierung einer Firma soziologisch nicht hinreichend verstehen - und damit letztlich auch praktisch-politisch nicht optimal behandeln. Wenn mit dem vorliegenden Beitrag die eingangs von J. A. Schumpeter ausgemachte terra incognita auch nur ansatzweise betreten worden ist, so ist andererseits eine Forschungsperspektive angedeutet worden, die lohnend erscheint, weiter verfolgt zu werden. Um zu verstehen, wie die „Akte“ der beruflichen Mobilität in das selbständige Unternehmertum sich im einzelnen vollziehen, muß die soziale Grammatik dieser beruflichen Veränderung im Spannungsfeld zwischen individuellem Anpassungsdruck und Freiwilligkeiten entschlüsselt werden. Die Frage nach der Komposition von Ressourcen kann auf der Mikroebene auch Hinweise darauf geben, warum Mobilität unterbleibt. Im Dechiffrieren dieser spezifischen sozialen „Logiken" dürfte übrigens gerade auch eine prinzipielle Stärke soziologischer Forschung im Vergleich zu anderen Disziplinen liegen.

\section{Literatur}

Aldrich, H., Rosen, B., Woodward, W., 1987: The Impact of Social Networks on Business Foundings and Profit: A Longitudinal Study. Paper presented at the 1987 Babson Entrepreneurship Conference, Pepperdine University at Malibu.

Barnes, J. A., 1954: Class and Committees in a Norwegian Island Parish, in: Human Relations, Vol. 7.

Bechhofer. F., Elliott, F. (Hrsg.), 1981: The Petite Bourgeoisie. Comparative Studies of the Uneasy Stratum, London-Basingstoke

Beck, U.. 1986: Die Risikogesellschaft. Auf dem Weg in eine andere Moderne, Frankfurt.

Biermann. B., 1971: Die soziale Struktur der Unternehmerschaft. Demographischer Aufbau, soziale Herkunft und Ausbildung der Unternehmer in NordrheinWestfalen, Stuttgart.

Blaschke, D., 1987: Erfolgswege zum neuen Arbeitsplatz, in: MittAB 2, 1987

Blau, P. M., 1956: Bureaucracy in Modern Society, New York.

Blossfeld, H. P., 1986: Career opportunities in the Federal Republik of Germany: a dynamic approach to the study of life-course, cohort and period effects, in: European Sociological Review, Vol. 2, No. 3.

Bögenhold, D., 1985: Die Selbständigen. Zur Soziologie dezentraler Produktion, Frankfurt-New York. 
Bögenhold, D., 1987 (a): Der Gründerboom. Realität und Mythos der neuen Selbständigkeit, Frankfurt-New York.

Bögenhold, D., 1987 (b): Selbständige im Beschäftigungssystem, in: A. Weymann (Hrsg.): Bildung und Beschäftigung, Soziale Welt, Sonderband 5, Göttingen.

Bögenhold, D., 1988: Die Selbständigkeit als Antwort. Empirische Resultate einer Großbetriebsstillegung, mimeo, Universität Münster.

Bourdieu, P., 1970: Klassenstellung und Klassenlage, in: ders.: Zur Soziologie der symbolischen Formen, Frankfurt.

Brinckmann, C., 1984: Die individuellen Folgen langanhaltender Arbeitslosigkeit. Ergebnisse einer repräsentativen Längsschnittuntersuchung, in: MittAB 4, 1984.

Burnham, J., 1941: The Managerial Revolution, New York.

Carroll, R. G., Mayer, K. U., 1986: Job-Shift Patterns in the Federal Republic of Germany: The Effects of Social Class, Industrial Sector, and Organizational Size, in: American Sociological Review, Vol. 51.

Chinoy, E., 1955: Automobil Workers and the American Dream, New York.

Daheim, J., 1960: Die Vorstellungen vom Mittelstand, in: Kölner Zeitschrift für Soziologie und Sozialpsychologie.

Dahrendorf, R., 1956: Industrielle Fertigkeiten und soziale Schließung, in: Kölner Zeitschrift für Soziologie und Sozialpsychologie.

Dahrendorf, R., 1957: Soziale Klassen und Klassenkonflikt in der industriellen Gesellschaft, Stuttgart.

Deeke, A., Fischer, J., Schumm-Garling, U. (Hrsg.), 1987: Arbeitsmarktbewegung als sozialer Prozeß, SAMF-Arbeitspapier 3-1987.

Forschungsgruppe Betriebsstillegung, 1989: Betriebsstillegung und Arbeitsmarkt. Die Folgewirkungen der Schließung der AG Weser, DFG-Endbericht, Universität Bremen.

Fröbel, J., 1874: Die Privatwirtschaft und die Volkswirtschaft, Leipzig.

Fürstenberg, F., 1962: Das Aufstiegsproblem in der modernen Gesellschaft, Stuttgart.

Geiger, T., 1962: Zur Theorie des Klassenbegriffs und der proletarischen Klasse (zuerst 1930), in: ders.: Arbeiten zur Soziologie, Neuwied.

Geiger, T., 1967: Die soziale Schichtung des Deutschen Volkes (zuerst 1932), Stuttgart.

Goffman, E., 1972: Asyle, Frankfurt.

Goldthorpe, J. H., Lockwood, D., Bechhofer, F., Platt, J., 1968: The Affluent Worker: Industrial Attitudes and Behaviour, London.

Goldthorpe, J. H., 1980: Social Mobility and Class Structure in Modern Britain, Oxford.

Goldthorpe, J. H., Payne, C., 1986: Trends in intergenerational class mobility in England and Wales 1972-1983, in: Sociology.

Granovetter, M. S., 1973: The Strength of Weak Ties, in: American Journal of Sociology, Vol. 78 (May).

Granovetter, M. S., 1974: Getting a Job. A study of Contacts and Careers, Cambridge.
Hanefeld, U., 1987: Das Sozio-ökonomische Panel. Grundlagen und Konzeption, Frankfurt-New York.

Hartmann, H., 1959: Authority and Organization in German Management, Princeton.

Hartmann, H., Eberlein, G., Unterfichter, S., 1968: Die Unternehmerin. Selbstverständnis und soziale Rolle, Köln-Opladen.

Haupt, H. G. (Hrsg.), 1985: Die radikale Mitte. Lebensweise und Politik von Handwerkern und Kleinhändlern in Deutschland seit 1848, München.

Jahoda, M., Lazarsfeld, P., Zeisel, H., 1969 (1933): Die Arbeitslosen von Marienthal. Ein soziologischer Versuch über die Wirkungen langandauernder Arbeitslosigkeit, Frankfurt.

Kaiser, M., Nuthmann, R., Stegmann, H. (Hrsg.): Berufliche Verbleibforschung in der Diskussion, Beiträge zur Arbeitsmarkt- und Berufsforschung, Nürnberg.

Lange, F. A., 1865: Die Arbeiterfrage in ihrer Bedeutung für Gegenwart und Zukunft, Duisburg.

Mayer, H. L., 1983: Umschichtung der Erwerbsbevölkerung. Bestands- und Längsschnittergebnisse des Mikrozensus, in: Wirtschaft und Statistik. Heft 10.

Mayer, K. U., 1977: Fluktuation und Umschichtung. Empirische Untersuchungen zu Strukturen sozialer Ungleichheit - Prozesse sozialer Ungleichheit in der Bundesrepublik Deutschland, Habil.-Schrift, Universität Mannheim.

Mayer, K. U., Carroll, G. R., 1987: Jobs and Classes: Structural Constraints on Career Mobility, in: European Sociological Review, Vol. 3, No. 1.

Mayer, K. U., 1988: Biographie oder Lebenslauf? Über die Tauglichkeit zweier Konzepte, Arbeitsskript, Fernuniversität-Gesamthochschule Hagen.

Michels, R., 1934: Umschichtungen in den herrschenden Klassen nach dem Kriege, Stuttgart.

Mill, J. S., 1848: Principles of Political Economy, London.

Mosakowski, M., Carroll, G. R., 1985: The Career Dynamics of Entrepreneurship: An Empirical Analysis of Self-Employment in the Federal Republik of Germany, mimeo, Max-Planck-Institut für Bildungsforschung, Berlin.

Müller, W., 1978: Klassenlage und Lebenslauf. Untersuchungen zu Prozessen sozialstrukturellen Wandels in der Bundesrepublik Deutschland, Habil.-Schrift, Universität Mannheim.

Müller, W., 1986: Soziale Mobilität: Die Bundesrepublik im internationalen Vergleich, in: Kaase, M. (Hrsg.): Politische Wissenschaft und politische Ordnung, Opladen.

Müller, W., Goldthorpe, J. H., 1988: Comparative Analysis of Social Mobility in Industrial Nations, Abschlußbericht, mimeo, Universität Mannheim.

Payne, G., 1987: Employment and Opportunity, London.

Polanyi, K., 1978: The Great Transformation, Frankfurt.

Preißendörfer, P., Voss, T., 1988: Arbeitsmarkt und soziale Netzwerke, in: Soziale Welt, 1, 1988.

Schelsky, H., 1968: Die Bedeutung des Klassenbegriffs für die Analyse unserer Gesellschaft (zuerst 1961), in: 
Seidel, B., Jenkner, S. (Hrsg.): Klassenbildung und Sozialschichtung, Darmstadt.

Schumpeter, J. A., 1953: Die sozialen Klassen im ethnisch homogenen Milieu (zuerst 1927), in: ders.: Aufsätze zur Soziologie, Tübingen.

Schumpeter, J. A., 1985: Unternehmerfunktion und Arbeiterinteresse (zuerst 1927), in: Stolper, W. F., Seidl, C., (Hrsg.): Joseph A. Schumpeter: Aufsätze zur Wirtschaftspolitik, Tübingen.

Schumpeter, J. A., 1964: Theorie der wirtschaftlichen Entwicklung, 6. Aufl., Berlin.

Schupp, J., 1988: Erwerbsbeteiligung und Arbeitsmarkt, in: H. J. Krupp und J. Schupp (Hrsg.): Lebenslagen im Wandel: Daten 1987: Frankfurt-New York.

Schütz, A., 1971: Gesammelte Aufsätze, Den Haag.

Schütz, A., Luckmann, T., 1975: Strukturen der Lebenswelt, Darmstadt.

Simmel, G., 1968 (1908): Soziologie. Untersuchungen über die Formen der Vergesellschaftung, 5. Aufl. Berlin.
Sombart, W., 1929: Gewerbewesen, 2. Aufl. BerlinLeipzig.

Urbat, F., 1974: Der Wechsel in die unternehmerische Selbständigkeit, Göttingen.

Veblen, T., 1924: Absentee Ownership and Business Enterprise in Recent Times, London.

Weber, M., 1972: Wirtschaft und Gesellschaft, 5. Aufl. Tübingen.

Weitzel, G., 1986: Beschäftigungswirkungen von Existenzgründungen, Gutachten i. A. des BMWi, München.

Wernet, W., 1952: Handwerkspolitik, Göttingen.

Windolf, P., Hohn, H. W., 1984: Arbeitsmarktchancen in der Krise. Betriebliche Rekrutierung und soziale Schließung, Frankfurt-New York.

Zapf, W. et al., 1987: Individualisierung und Sicherheit. Untersuchungen zur Lebensqualität in der Bundesrepublik Deutschland, München. 